\title{
L. Cossonius L. f. Stell(atina tribu) Gallus Vecilius Crispinus Mansuanius Marcellinus Numisius Sabinus pro consule provinciae Sardiniae e la constitutio del Forum Traiani*
}

\author{
Attilio Mastino \\ Università degli Studi di Sassari \\ mastino@uniss.it \\ Raimondo ZuCCA \\ Università degli Studi di Sassari \\ raimondoz@virgilio.it
}

Recibido: 20 de agosto de 2013

Aceptado: 20 de marzo de 2014

\section{RIASSUNTO}

Lo studio analizza la carriera del clarissimus L. Cossonius Gallus sviluppatasi fra Domiziano, Nerva, Traiano e Adriano. In particolare si analizzano le cariche sotto Traiano, fra le quali, nel 111 d.C., il proconsolato della Sardinia. Nello stesso anno il proconsole L. Cossonio Gallo per ordine di Traiano procedette alla constitutio di Forum Traiani, al centro della Sardinia. Successivamente Cossonio divenne nel 113-115 legatus Augusti delle provinciae della Galatia, Pisidia e Paphlagonia, nel 116 fu consul suffectus, e verso il 120 legatus provinciae Iudeae.

Parole chiave: L. Cossonius Gallus. Traiano. Sardinia. Fondazione di Forum Traiani. Galatia, Pisidia e Paphlagonia. Provincia Iudaea.

\section{Cossonius L. f. Stell(atina tribu) Gallus Vecilius Crispinus Mansuanius Marcellinus Numisius Sabinus pro consule provinciae Sardiniae and the constitutio of Forum Traiani}

\begin{abstract}
The study analyzes the career of clarissimus L. Cossonius Gallus developed between Domitian, Nerva, Trajan and Hadrian. In particular, we analyze the charges under Trajan, including, in $111 \mathrm{AD}$, the proconsul of Sardinia. In the same year the proconsul L. Cossonius Gallus by order of Trajan proceeded to constitutio of Forum Traiani in the center of Sardinia. Subsequently (113-115 AD) Cossonius became legatus Augusti in the provinciae of Galatia, Pisidia and Paphlagonia, was consul suffectus in 116 AD, and around 120AD legatus provinciae Iudeae.
\end{abstract}

* Il testo, benché concepito unitariamente, è dovuto ad Attilio Mastino $(\S 1,3,4,7)$ e a Raimondo Zucca $(\S 2,5,6)$. 
Key words: L. Cossonius Gallus. Trajan. Sardinia. Founding of Forum Traiani. Galatia, Pisidia and Paphlagonia. Provincia Iudaea.

Sumario: 1. La provincia Sardinia nel quadro dell'amministrazione senatoria e dell'amministrazione equestre fra l'età augustea e il periodo traianeo. 2. L. Cossonius L. f. Stell(atina tribu) Gallus Vecilius Crispinus Mansuanius Marcellinus Numisius Sabinus. 3. L. Cossonius Gallus proconsul Sardiniae. 4. La constitutio del Forum Traiani in Sardinia. 5. Topografia di Forum Traiani. 6. La carriera di L(ucius) Cossonius Gallus dopo il proconsolato della provincia Sardinia.7. Conclusioni.

\section{La provincia Sardinia nel quadro dell'amministrazione senatoria e dell'ammi- nistrazione equestre fra l'età augustea e il periodo traianeo}

La provincia Sardinia et Corsica, istituita nel 227 a.C. insieme alla provincia Sicilia, venne inizialmente lasciata da Augusto all'amministrazione senatoria (sotto la responsabilità di proconsoli ex pretori), in quanto considerata pacata. ${ }^{1}$ Solo nel 6 d.C., in occasione del grave bellum in Sardinia, presumibilmente in forza di un senatus consultum, si dové procedere a trasferire "per alcuni anni"' l'amministrazione delle due isole tirreniche dal senato all'imperatore, ${ }^{3}$ che provvide ad inviarvi un pro legato con forze armate ausiliarie (cohors I Corsorum, cohors Ligurum, cohors III Aquitanorum, cohors Lusitanorum $)^{4}$ forse rinforzate per l'occasione.

Al pretoriano(? $)^{5}$ T. Pompeius Proculus che è qualificato come obtinens (provinciam) con il titolo di pro legato nel miliario augusteo del 13-14 d.C. ${ }^{6}$ del X miglio della via (ad Forum Augusti?) (Austis, presso Fordongianus), ${ }^{7}$ successe il praefectus provinciae (Sardiniae) al principio dell'età di Tiberio. ${ }^{8}$ La provincia venne mantenuta nell'amministrazione imperiale fin quasi al termine del principato di Nerone. $\mathrm{Fu}$ Nerone, infatti, nel 67 d.C., dopo avere annunziato l'eleutheria della provincia

1 Mastino 2009, 116-121, 125-127 e 157-160. I fasti proconsolari della Sardinia fra il 27 a.C. e il 6 d.C. annoverano esclusivamente Q. Mucius Scaevola, [Q.? C]aecilius M[etellus Creticus] e [M. Fu]rius Ca[millus] (AE 1999, 804a) identificabile probabilmente con M. Furius P. f. P. n. Camillus, che dovette ottenere il proconsolato pretorio della Sardinia et Corsica verso il 5 o il 6 d.C. (Meloni 1958,183, nr. 1; Thomasson 2009, 3-4, nrr. 1-2; Zucca 2001, 516-527; Mastino - ZucCA, c.s.).

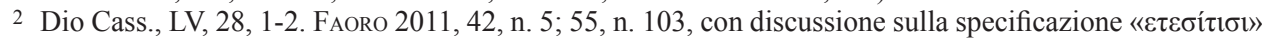

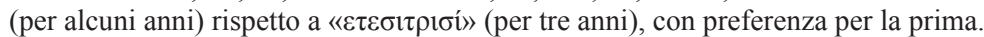

3 Mastino 2009, 125-127; FAORO 2011, 41-74.

4 Le Bohec 1990; IbBa 2009, 393-404; Mastino - ZucCA, c.s., con bibl. precedente.

5 Ipotesi proposta da FAORO 2011, 58.

6 Eph. Ep. VIII, 742 = ILS 105.

7 Sul possibile Forum Augusti desunto dal poleonimo medievale Agustis (odierna Austis), ipotizzato da Massimo Pittau e da Attilio Mastino cfr, da ultima, Crespo CABILlo 2009, 289.

8 Secondo FAORO 2011, 73-74, Tiberio avrebbe ottenuto un nuovo senatum consultum, ratificato da una lex a populo, relativo al passaggio della Sardinia dalle provinciae senatorie e quelle dell'imperatore, in forza del suo imperium proconsulare esercitato in Sardinia attraverso un praefectus provinciae equestre, attestato la prima volta nella targa di un edificio dedicato a Tiberio dalla civitates Barbariae in Aquae Ypsitanae (Fordongianus), all'inizio del principato di Tiberio. L'ipotesi è assai acuta in attesa di nuovi documenti chepossano asseverare l'ipotizzata trafila legislativa. Su differenti posizioni LoRETo 2000. 


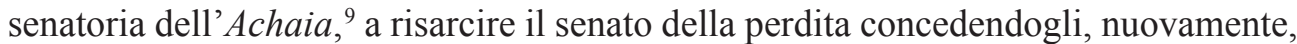
la Sardinia. ${ }^{10}$ Il ritorno della Sardinia all'amministrazione imperiale fu attuato da Vespasiano, probabilmente prima del 74 d.C., forse contemporaneamente alla restituzione dell'Achaia al senato. ${ }^{11}$

La dottrina ha riconosciuto il ritorno della Sardinia all'amministrazione senatoria sotto il principato di Traiano sulla base della documentazione epigrafica. ${ }^{12}$

Geza Alföldy nel 1991 ha proposto di attribuire ad età traianea, fra il 110 e il 117 d.C., ${ }^{13}$ precisata al 115 d.C. da Ch. Settipani, ${ }^{14}$ il pro consule provinciae Sardiniae C. Asinius Tucurianus,${ }^{15}$ di contro alla cronologia augustea, ante 6 d.C., ipotizzata da Oliver ${ }^{16}$ e successivamente da Thomasson. ${ }^{17}$

Con certezza assegniamo al periodo tardo traianeo il proconsolato della provincia Sardinia di L. Cossonius L. f. Stell(atina tribu) Gallus Vecilius Crispinus Mansuanius Marcellinus Numisius Sabinus. ${ }^{18}$

L'attestazione del proconsolato della Sardinia di Lucio Cossonio Gallo è presente in due testi epigrafici:

\section{1) Antiochia Pisidiae}

[L(ucio?) Cosso]nio(?) L(uci) f(ilio) Stel(latina) / Gallo Vecilio / Crispino Mansuanio / Marcellino Numisio / [S]abino leg(ato) Aug(usti) pro pr(aetore) / provinciar(um) Galatiae Pisid(iae) / [P]aphlagoniae sodali Fla/viali proco(n)s(uli) prov(inciae) Sard(iniae) / leg(ato) legionum I Italicae et / [I]I Traianae Fortis praef(ecto) frum(enti) / dandi curatori viar(um) Clodiae / Cassiae Anniae Ciminae Tra/ianae Novae praetori

9 L'Achaia fu costituita come provincia autonoma senatoria nel 27 a.C. (Dio Cass., LIII, 12; Strab., XVII, p. 840). Tiberio, nel 15 d.C. riunì l'Achaia alla Macedonia, soggetta all'amministrazione imperiale del legatus Augusti propraetore. Solo nel 44 d.C. Claudio ricostituì la provincia di Achaia riassegnandola al senato (Tacit., Ann., I, 76; Dio Cass., LVIII, 24, LX, 24; Sueton., Claud., 25).

10 Sueton., Nero, 24; Plut., Flamin., 13; Plin., Nat. Hist., IV, 22; Dio Cass., LXIII, 11; Pausan., VII, $17,2$. I fasti dei proconsules Sardiniae nel periodo 67-74 d.C. annoverano: (Cn.) Caecilius Simplex (67/68 d.C.), L. Helvius Agrippa 68/69 d.C.), [---]atius Secundus (70 d.C.); cfr. Meloni 1958, 34-5, nrr. 8-9, 11; Mastino 2009 , 116-121; ZuCCA 2001, 528, nrr. 10-12. Sono noti per il proconsolato di Lucio Elvio Agrippa un legatus pro praetore, M. Iulius Romulus, ed un quaestor pro praetore, T. Atilius Sabinus (CIL X $7582=$ ILS 5947). Incerto è il proconsole, fra il 67/68 e il 70-73 d.C., cui dovrà assegnarsi il pr(o)legat(o) pr(o) pr(aetore) provinciae Sardiniae dell'iscrizione di Sestinum CIL XI 6009 = ILS 981: C(aio) Caesio T(iti) f(ilio) Cl(ustumina) / Apro praef(ecto) coh(ortis) / Hispanor(um) equi/tatae, trib(uno) milit(um), / quaestori pro pr(aetore) / Ponti et Bithyniae, / aedili pleb(is) Cer(iali), pr(o) / legat(o) pr(o) pr(aetore) pro/vinciae Sardiniae / d(ecreto) decurionum). Sul personaggio cfr. Mastino in Pais 1999, 43, n. 116; Mastino - Ruggeri 1998, $243-244$.

11 Mastino 2009, 143; FaORo 2011, 307.

12 Mastino 2009, 145.

13 Alföldy 1992, 131-135; EcK - Heil 2005; Feraudi-GruÉnAis 2005, 148, n. 30; Alföldy in CIL VI 8, II, p. 4379, 4321, nota.

14 SetTipani 2000, 266-267; ID. 2002, 22, 100.

15 PIR A 1254; RE II, c. 1604, nr. 38.

16 Oliver 1947, 159, n. 27; Dietz 1980, 88-89, n. 215.

17 Thomasson 2009, 4, nr. 2:44: "Mihi prius sub Augusto ponendus videbatur; contra Alföldy, G. (1992), 137-139, potius de ultimis annis imperatoris Traiani cogitare videtur, aetatem Augusti utique excludens".

18 PIR ${ }^{2}$ G 71; PIR C 1541; RE I, cc. 2268-9, nr. 51; DNP 12/2, 934; MELONi 1958, 198-200, nr. 19; ECK 1970, 41, 46-47, 178, 180, 184-6; JACQUes 1983,_80-81, n. 5; ThOMASson 2009, 4, nr. 2:016. 
trib(uno) pl(ebis) / quaestori provinc(iae) Ponti et / [B]ithyniae leg(ato) Asiae, III$\operatorname{vir}\left(\right.$ o) capital(is), / trib(uno) mili/t(um) leg(ionis) XXI Rapacis. ${ }^{19}$

2) Horbat Qesari / Caesarea Maritima

[L(ucio) Cosso]nio L(uci) f(ilio) S[tel(latina) Gallo] / [Vecil]io Cris[p]ino Ma[nsuanio] / [Marc]ellino Numi[sio Sabino] / [co(n)s(uli) VI]Ivir(o) epul(onum) [legato Imperatoris Hadri]/[ani Aug(usti) p]r(o) pr(aetore) [p]rovin[ciae Iudaeae, leg(ato) pr(o)] / [pr(aetore) prov(inciae) Gal]atiae, pro[co(n)s(uli) prov(inciae) Sard(iniae)], I [leg(ato) leg(ionis) I Itali(cae)] et leg(ionis) I[I Traian(ae) Fort(is) ] / _ . . _ _20

\section{L. Cossonius L. f. Stell(atina tribu) Gallus Vecilius Crispinus Mansuanius Mar- cellinus Numisius Sabinus}

Lucio Cossonio è di origine italica, benché ignoriamo il luogo d'origine della gens. L'ascrizione alla tribù Stellatina di L. Cossonius Gallus e l'alleanza matrimoniale con gli Eggi Marulli di Aeclanum ${ }^{21}$ ci induce a credere che la città d'origine della gens potesse essere Beneventum, distante XV m.p. da Aeclanum e i cui cives erano in prevalenza ascritti alla Stellatina. ${ }^{22}$ La polionimia di Lucio Cossonio Gallo, d'altro canto, ci rimanda alle gentes, appartenenti all'ordine senatorio, Vecilia, ${ }^{23}$ Mansuania $^{24} \mathrm{e}$ Numisia. ${ }^{25}$ Sul suo ingresso in Senato non si possiedono dati, per cui può pensarsi ad un accesso recenziore della gens Cossonia. ${ }^{26}$

La carriera di Lucio Cossonio Gallo debutta, verso il 90-92 d.C.(?), con lo svolgimento del tribunato militare laticlavio nella legio XXI Rapax, distrutta dai Sarmati nel 92 d.C., ${ }^{27}$ ovvero, meno probabilmente, sciolta da Domiziano per avere appoggiato la rivolta di L. Antonius Saturninus nell'89 d.C. ${ }^{28}$

In Roma Cossonio svolge, nell'ambito del vigintivirato, la magistratura di triumvir capitalis, probabilmente intorno al 93 d.C. ${ }^{29}$

Successivamente riveste gli incarichi provinciali di legatus della provincia proconsolare d'Asia e di quaestor della provincia di Pontus et Bithynia. Rientrato a Roma

19 CIL III $6813=I L S 1038=A E 1888,90$.

$20 C I I P, 2,1227=A E 2003,1801$.

21 CAMOdeCa 1982, 132-134; $P I R^{2}$ E 10.

22 Torelli 2002, 151-154.

23 Cébeillac-Gervasoni 1982, 73 (incerto).

24 AE 1998, $1131=A E$ 1999, 93b. Novae (Moesia inferior): [A] esculapium / ex donis arg(enteum) / p(ondo) V unc(iis) V/C(aius) Mansuanius / Severus leg(atus) Aug(usti) f(aciendum) c(uravit). Il legatus della I Italica C(aius) Mansuanius Severus non può comunque identificarsi con L. Cossonius Gallus. Cfr. EcK 2013a. La gens è di rara attestazione: CIL VI 22011 (p. 3528) (Roma); CIL XI 672 (Aemilia, loc. Imola / Forum Cornelii); AE 1957, 138 (Aemilia, loc. Boncellino di Bagnacavallo); AE 2003, 662 (Aemilia, loc. Castelfranco Emilia / Mutina); CIL II 576 = ERAE $325=$ HEp 13, 2003-2004,167 (Lusitania, Augusta Emerita).

25 TORELLI 1982, 254, 265-267, 278.

26 Cfr. McAlindon 1957, 191-193.

27 Ritterling 1893, 233; ID. 1925a, 1736, 1788-9; Syme 1936, 177; Mócsy 1974, 83-84; Strobel 1986, 217; ID. 1988, 216; ID. 1989, 84 e passim; Wilkes 1983, 269-70; Jones 2002, 149, 152; UrLoIU 2010. Le fonti antiche sono costituite da Suetonius, Domitianus, VI, 1, ed Eutropius, VII 23, 4.

28 BÉRARd 2000, 49-67.

29 Cascione 1999, 233-234; Hillebrand 2006, 92-94, 250-251, nr. 15 (prosopographia). 
diviene dapprima tribunus plebis e quindi praetor. Seguono, sempre nell'Urbe, la curatela delle viae Clodia, Cassia, Annia, Cimina e Traiana Nova e la prefettura frumenti dandi.

L'unico dato cronologico nella sequenza di questi sei ruoli è costituito dal terminus post quem del 108 per la cura viarum, in quanto in quell'anno Traiano viam novam Traian(am) a Volsinis ad fines Clusinorum fecit, ${ }^{30}$ secondo il dettato dei due miliari traianei del 108 d.C. relativi al miglio XIII ${ }^{31}$ ed al miglio XVII..$^{32}$

Cossonio divenne, quindi, legatus della legio I Italica,${ }^{33}$ costituita da Nerone, stanziata da Vespasiano a Novae (Svishtov, Bulgaria), ${ }^{34}$ e utilizzata da Traiano nel bellum dacicum $^{35}$ e successivamente legatus della legio II Traiana Fortis istituita da Traiano, probabilmente dopo la II dacica, ${ }^{36} \mathrm{e}$ stabilita nelle regioni danubiane, forse in Moesia a Novae, da cui fu trasferita in Iudaea.

La cronologia più probabile per il comando delle due legioni, da parte di Cossonio, è verso il 109-110 d.C.

\section{L. Cossonius Gallus proconsul Sardiniae}

Dopo i comandi legionari Lucio Cossonio ebbe il proconsolato della Sardinia probabilmente intorno al 111 d.C. ${ }^{37}$

30 Garrucci 1864, 7-8; Moretti 1925; Harris 1965, 113-133; Bennett 2005. La via nova Traiana corrisponde, insieme alle altre viae coherentes alla Cassia, alle viae tres Traianae. Per alcuni esempi: nova Traiana: CIL V 877 (p. 1025) =ILS 1052 (p. 173) =InscrAqu.1, $498=$ IEAquil $65=A E$ 1959, $155=A E$ 1995, 571; CIL VI $1356=$ ILS 1109; tres Traianae: CIL III $7394=$ III $12324=$ ILS 1093; CIL IX $5883=$ ILS 1059 = CRISTOFori 2004, 166-175; AE 1927, 77; viae Traianae: Corinth VIII, 3, nr.125. Secondo Thomasson 2009, 103, nr. 28:019 Lucio Cossonio "curator viae novae Traianae fuit non ante a. 109 (RE Suppl. XIII 1641, 33-54)".

31 CIL XI 8104 = ILS 9496: Imp(erator) Caes(ar) / divi Nervae f(ilius) / Nerva Traianus / Aug(ustus) Germ(anicus) Dacic(us) / pont(ifex) max(imus) trib(unicia) p(otestate) XII / imp(erator) VI co(n)s(ul) V p(ater) p(atriae) / viam novam Traian(am) / a Volsinis ad fines / Clusinorum fecit / XIII.

$32 A E$ 1926, 112. Regio VII, loc. Fabro: Imp(erator) Caesar / divi Nervae f(ilius) / Nerva Traianus / Aug(ustus) Germ(anicus) Dacicus / pont(ifex) max(imus) trib(unicia) pot(estate) XII / [i]mp(erator) VI co(n)s(ul) V p(ater) p(atriae) / viam novam [Tra]ia[n]/a(m) Volsinis ad fines / Clusinorum fecit / XVII. Il miliario è palinsesto: $A E$ 1926, $113=A E$ 1969/70, 185b = AE 2002, 463 (HARRIs 1991, 186-188).

33 RitTerling 1925, 1407-1417.

34 SARNOWSKI 2001. Per le fonti epigrafiche: CIL III 750 (p. 992, 1338); 6232, 6239a; 7438, 7441; 7617; $A E 1932,53 ; 1937,97 ; 1944,14 ; 1965,134 ; 135 ; 1966,345 ; 1968,454 ; 1972,526,528 ; 1973,480 \mathrm{a}-\mathrm{b} ; 1975$, 754; 1982, 849; 1983, 878; 1987, 866b; 1985, 735; 1987, 863; 1988, 984; 1990, 863; 1991, 1374; 1993, 1364a; 1995, 1334; 1996, 1339 e 1340; 1998, 1130,1132 e 1133; 1999, 1332, 1333 e 1335; 2004, 1243.

35 CIL VI 3584 e CIL XI 3100

36 Sir Ronald Syme ritenne assai improbabile la partecipazione della II Traiana Fortis alle guerre daciche (Syme 1938=1971, 91; Urloiu 2010, 4); contra Strobel 1988a, 271-273, che propone datazioni troppo risalenti per la costituzione della legio.

37 EcK (2013) ha proposto la data del 111 per il proconsolato della Sardinia. Thomasson 2009, 103, nr. 28:019, propone una forbice più ampia fra il 110-114 d.C. o anche successivamente (procos. Sardiniae fortasse quo tempore provinciae Bithyniae praefuerunt legati Augusti pro praetore (c. 110-114, vel etiam postea)). Importante è l'osservazione di ECK $(2005,62)$ relativa alla evidenza di COS in procos. Sardiniae della iscrizione CIL III $6813=$ ILS 1038, per rimarcare il rinnovato ruolo senatorio nell'amministrazione della Sardinia. 
La scelta del mutamento amministrativo della Sardinia con il ritorno della stessa provincia al Senato, dovette pendere, certo, da una decisione di Traiano, ${ }^{38}$ ma determinata de iure da un senatus consultum, che, a ragione, si è ipotizzato anche per il primo passaggio della Sardiniapost 6 d.C. dal Senato all'amministrazione dell'Imperatore. ${ }^{39}$

In una ferrea logica di variazioni amministrative provinciali ci appare plausibile ritenere che la Sardinia passasse al Senato come bilanciamento del transito della provincia di Ponthus et Bithynia ${ }^{40}$ dall'amministrazione senatoria a quella imperiale, forse nel 111 d.C. ${ }^{41}$

La celebre iscrizione comasca di Plinio il Giovane documenta infatti che Plinio divenne legat(us) pro pr(aetore) provinciae Pon[ti et Bithyniae pro]/consulari potesta[t(e)] in eam provinciam e [x s(enatus) c(onsulto) missus ab] / Imp(eratore) Caesar(e) Nerva Traiano Aug(usto) German[ico Dacico p(atre) p(atriae)]. ${ }^{42}$

38 Mastino 2009, 145. Il periodo di amministrazione senatoria della Sardinia dovette coprire quasi integralmente il II secolo d.C., fino a Settimio Severo, allorquando, verso il 193, compare nuovamente un procurator et praefectus provinciae Sardiniae. I fasti della provincia senatoria della Sardinia del II sec. sono estremamente lacunosi: 1) L(ucius) Cossonius L(uci) f(ilius) Stel(latina) Gallus Vecilius Crispinus Mansuanius Marcellinus Numisius Sabinus. 2) C. Asinius Tucurianus (PIR A 1254; RE II 1604, n. 38). 3) M'rqh Phedwq'yh Pl'wty [---] identificabile con M. Peducaeus Plautius Quintillus (proco(n)s(ul) prov(inciae) Sard(iniae))? (169-176 d.C.). Cfr. ZucCA 2001, 528, n. 70; per l'iscrizione Levi Della Vida 1934-1935, 185-198; Février 1946-1949, 415-419; Guzzo Amadasi 1967, 133-136, Sardegna, n. 8 Npu. 4) L. Ragonius Urinatius Larcius Quintianus (PIR ${ }^{2}$ R 17; RE I A, cc. 128-129, n. 5 -Extremis Marci Aurelii annis (c. a. 175?).-procos. prov. Sardin. CIL VI 1502 (cf. add. pp. 4706 sq.) = ILS 1124; pro cos. provinc. Sard. CIL V 2112, Tarvisium (MeLonI 1958, 200-201, nr. 20). Durante il periodo di governo senatorio del II sec., a parte la quaestura rivestita da Settimio Severo, il futuro imperatore (Ael. Spart. Vita Severi 2), sono noti due probabili procuratores finanziari, ritenuti da altri procuratores et praefecti provinciae Sardiniae: 1) Claudius Paternus Clementianus (PIR ${ }^{2}$ C 953; RE III 2840 sq., n. 262). (Thomasson 2009, 6, nr. 02:46: "Traianus-Hadrianus (?). - proc. Aug. provincia[rum] Iudaeae Sar[diniae] Africae et [Norici] 121 (ILS 1369), Abudiacum. Meloni 1958, 196-7, nr. 17; H.-G. Pflaum, Carrières I 354-358 (necnon III 978), n. 150 bis, cf. Suppl., 43. Quod ad aetatem huius viri attinet, H.-G. Pflaum, 1. c., monuit (1) civitatem Romanam avo materno eius datam esse a Claudio vel Nerone imperatoribus, (2) eum haud ante a. 85 praefectum alae Silianae torquatae civium Romanorum fuisse, (3) procuratorem autem provinciae Iudaeae eum fuisse ante a. 135, a quo anno ea provincia Syria Palaestina appellata sit"). Per l'interpretazione come procurator finanziario della provincia senatoria cfr. ZucCA 2001, 528; Faoro 2011, 285, n. 97. 2) [pr]oc. Augg. Mastino 1986, 10, nr. $1=$ AE 1992, 905, Turris (Thomasson 2009, 6, nr. 02:50 a (haud ante Marcum)). Ginette di Vita-Evrard ha proposto come data il 166 d.C. in $A E$.

39 FAORO 2011, 73-74. Naturalmente anche il passaggio della Sardinia dall'Imperatore al Senato nel 67 d.C., per decisione di Nerone, e il suo ritorno all'Imperatore sotto Vespasiano dovette essere sanzionato da due distinti senatus consulta.

40 Per la dubbia cronologia, dipendente dalla datazione delle epistulae del libro X di Plinio il Giovane, cfr. da ultimo BENNETT 2005, 118: "Unfortunately, although we learn from the Letters that Pliny arrived in his province on 17 September, there is no indication of which year. Various internal references, however, allow it to be deduced as either 109, 110 or 111, and the Letters end abruptly with Pliny's death before two full years had elapsed (Williams, W. (1990), 13, for a discussion). As A.N. Sherwin-White has observed, the year 109 seems most likely for his appointment as in Letter 100, the second and last New Year's address to the emperor, Pliny does not register the consulship Trajan took up on 1 January 112 (SHERwin-White, A. N. (1966), 81) In support of the claim, we might also adduce Pliny's failure to register Trajan's designation as consul in the elections of late 111, and also the lack of any reference to the death of Plotina on 29 August 112". Vedi anche CASTAGNA - LefĖvre 2003 con la proposta cronologica $110 / 112$ d.C.

41 ECK 1970, 13; MareK 2003; WeHManN 2008, 75, n. 427.

$42 C I L$ V $5262=C I L$ VI, p. $4712=I L S 2927$ (p. 180) $=A E 1947,65=A E 1963,190=A E 1966,127=$ $A E$ 1983, $444=A E$ 1984, $436=A E$ 1999, $747=A E$ 2001, 1085. Regio XI, Comum. C(aius) Plinius L(uci) 
Indubbiamente il senatus consultum evocato dall'iscrizione comasca e dalla seconda epigrafe relativa a Plinio il Giovane da Hispellum non implica il passaggio definitivo della provincia del Pontus et Bithynia al Senato, poiché se è vero che il successore di Plinio in quella provincia, C. Iulius Cornutus Tertullus ${ }^{43}$ è ugualmente legatus pro praetore divi Traiani [Parthici] / provinciae Ponti et Bith[yniae] ${ }^{44} \mathrm{e}$ nel tardo principato adrianeo C. Iulius Severus è un legatus ad corrigendum statum provinciae (Cass. Dio 69,14,4), solamente sotto Antonino Pio Pontus et Bithynia ottengono definitivamente lo status di provincia imperiale. ${ }^{45}$

La Sardinia era stata amministrata da procuratores et praefecti provinciae, di rango equestre, dal 74 d.C. Indubbiamente ancora nel primo decennio del principato di Traiano la provincia era mantenuta dall'imperatore: se un miliario frammentario relativo alla restitutio del XVI miglio (loc. Tanì-Carbonia) ${ }^{46}$ della via quae a Sulcis Karales ducit ${ }^{47}$ non ha documentato il nome del procurator et praefectus della

f(ilius) Ouf(entina) Caecilius [Secundus co(n)s(ul)] / augur legat(us) pro pr(aetore) provinciae Pon[ti et Bithyniae pro]/consulari potesta[t(e)] in eam provinciam e[x s(enatus) c(onsulto) missus ab] / Imp(eratore) Caesar(e) Nerva Traiano Aug(usto) German[ico Dacico p(atre) p(atriae)] / curator alvei Ti[b]eris et riparum e[t cloacar(um) urb(is)] / praef(ectus) aerari Satu[r]ni praef(ectus) aerari mil[it(aris) pr(aetor) trib(unus) pl(ebis)] / quaestor Imp (eratoris) sevir equitum [Romanorum] / trib(unus) milit(um) leg(ionis) [III] Gallica[e Xvir stli]/tib(us) iudicand(is) therm [as ex HS] adiectis in / ornatum HS CCC(milibus) [ et eo amp]lius in tutela [m] / HS CC(milibus) t(estamento) f(ieri) i(ussit) [item in alimenta] libertor(um) suorum homin(um) $C$ / HS XVIII(centena) LXVI(milia) DCLXVI rei [p(ublicae) legavit quorum inc]rement(a) postea ad epulum / [p]leb(is) urban(ae) voluit pertin[ere ...]s dedit in aliment(a) pueror(um) / et puellar(um) pleb(is) urban(ae) $H S[D$ (milia) et] in tutelam $b<i=Y>$ bliothe/cae $H S C$ (milia). Il rango di legat(us) pro pr(aetore) provinciae Pon[ti et Bithyniae] per Plinio il Giovane, parimenti, documentato nel titulus di Hispellum CIL XI $5272=C I L$ VI 1552 (p. 3805, 4712) = AE 1892, $5=A E$ 1999, $92=A E$ 1999, 612. Regio VI. Hispellum: [C(aius) Plinius L(uci) f(ilius) Ouf(entina) Caecilius Secundus co(n)s(ul) augur] / [Xvir stlit(ibus) iudicand(is) trib(unus) mil(itum) leg(ionis) III Galli]ca[e] / [sevir eq(uitum) R(omanorum) quaestor Imperatori]s trib(unus) plebis pr(aetor) / [praef(ectus) aer(arii) milit(aris) praef(ectus) aer(arii) Saturni cur(ator) alvei] Tiberis ex s(enatus) c(onsulto) pro/[consulari potestate legatus pr(o) pr(aetore) provinciae Ponti] et Bithyniae et legatus / [in eam ab Imp(eratore) Caes(are) Nerva Traiano Aug(usto) missus testame]nto [[[fieri]]] iussit. Appare estremamente plausibile l'integrazione di Geza Alföldy [pro]/consulari potesta[t(e)] nell'iscrizione di Como e pro/[consulari potestate] in quella di Hispellum, che riprende una feconda ipotesi di Bormann, di contro alla forma consulari potestate ammessa dal Mommsen in poi (ALFöLDY 1999, 21-44; ID. 1999a, 221-244; ECK 2001, 225- 235; ID. 2009, 85-86, n. 25).

$43 P^{2} \mathrm{~J} 273,706$.

44 CIL XIV 2925 = ILS $1024=$ EE, 9, p 432. Tusculum: C(aio) Iulio P(ubli) f(ilio) Hor(atia) [3] / Cornuto Tertul[lo] / co(n)s(uli) proconsuli provinci[ae Asiae] / proconsuli provinciae Narbo[nensis] / legato pro praetore divi Traiani [Parthici] / provinciae Ponti et Bith [yniae] / eiusdem legato pro pr[aetore] / provinciae Aquitani[ae] c[e]nsu[um] / accipiendorum cu[ra]to[ri viae] / Aemiliae praefecto aerari Sa[tu]r[ni] / legato pro praetore provinc[iae] / Cretae et Cyrenarum [3] a[dl]e[cto] / inter praetorios a divis Ves[pasiano] / et Tito censoribus aedili ce[riali] / quaestori urbano / ex testamento / C(aius) Iulius Plan $\{i\}$ cius Varus Cornutus [- - - ].

45 Strobel 2013.

46 Atzori 2006, 140-141, nr. 9.

ILSard I 373=ELSard A. 373. Cfr. Atzori 2006, 140-141, nr. 9: XVI (M. P.) / Imp(erator) Caesar divi / Nervae f(ilius) Nerva / Traianus Aug(ustus) / op(timus), Dacic(us), p(ontifex) m(aximus) (104 d.C.; nuova lettura di A. Ibba, M. Sechi, A. Gavini, B. Cocco e T. Ganga del 26 luglio 2013). Per il titolo optimus votato dal Senato a Traiano nell'estate del 114 d.C. cfr. BenNett 2005, 106-119, 198, n. 60. Nel miliario è possibile sciogliere anche o(ptimus) $p$ (rinceps).

47 La denominazione della via è ricostruita ipoteticamente in base al miliario traianeo CIL X 8004. Secondo i milliari ILSard I 371, 372, 373 il caput viae era Sulcis. Per il miliario CIL X 8006 con la denominazione 
Sardinia, ed un diploma del 102 d.C. restituisce uno spezzone infimo del gentilizio del governatore della Sardinia ${ }^{48}$ un secondo miliario traianeo ${ }^{49}$ della stessa via a Sulcis del 106 d.C. da Sant' Andrea (Assemini) attesta come governatore provinciale l'equestre [C.] Ulpi[us Se]v[erus proc(urator) Aug(usti) praef(ectus) pro]vi[nciae Sard(iniae) $]. .^{50}$ Probabilmente allo stesso 106 d.C. o agli anni immediatamente precedenti o seguenti deve assegnarsi la dedica [Di]ana[e et ] Silvano [n]emoris Sorabensis, il bosco sacro presso la statio di Sorabile della via ab Vlbia Caralis per mediterranea, posta dal procurator et praefectus C. Ulpius Severus. ${ }^{51}$

Il primo proconsul Sardiniae di età traianea sembra essere proprio Lucio Cossonio Gallo, poiché Gaio Asinio Tucuriano, anche nella proposta cronologica di Geza Alföldy parrebbe assegnabile al 110/115 d.C.

della via Karalibus Sul[cos] e per la statio di ad decimum milliarium (attuale Decimomannu) il caput viae era Karales. Cfr. MAstino 2009, 382-383 e Atzori 2006.

48 Pala - Sanciu - Sanges 2013, 301-306: Intus: [Imp(erator) Caesar divi Nervae] f(ilius) Nerva Traianus Au/[gustus Germanicus p]ontifex maximus / [tribunic(ia) potestat(e)] VI imp(erator) II co(n)s(ul) IIII p(ater) p(atriae) / [peditibus et equitibus q]ui militaverunt in / [cohortibus duabus qu]ae appellantur I Gemi/[na Sardorum et Cors]orum et II Gemina Li/[gurum et Corsorum] et sunt in Sardinia / [sub - - -]o quinis et vicenis / [pluribusve stipendiis] emeritis dimissis / [honesta missione]quorum nomina / [subscripta sunt ipsi]s liberis posteris/[que eorum civitat]em dedit et conu/[bium cum uxoribu]s quas tunc habu/[issent cum est civi] tas iis data aut / [siqui caelibes essen] t cum iis quas / [- - ] Extrinsecus: [- - ] quas postea duxissent dumta[xat sin]/guli singulas. A(nte) d(iem) III Non(as) Maias / T. Didio Secundo / L. Publilio Celso / co(n)s(ulibus) / cohort(is) IIGeminae Ligurum et Corsorum / cui prae(e)st L\{u\}cius Terentius Serenus / ex pedite / Hannibali Tabilatis f(ilio) Nur(-) Alb(-) / et Iurini Tammugae filiae uxori eius Sordia / et Sabino f(ilio) eius / et Saturnino f(ilio) eius / et Tisareni filiae eius / et Bolgittae filiae eius / et Bonassoni filiae eius. / Descriptum et recognitum ex tabula ae/nea quae fixa est Romae in muro post / templum divi Aug(usti) ad Minervam. In accordo con una delle ipotesi dei tre editori si concorda nel ritenere letterario il nome Hannibal e non retaggio dell'antroponimia punica diffusa in Sardegna ancora in età romana. Per gli altri nomi appare chiaro l'ambito encorico di pertinenza (sottolineato dagli autori dell'edizione del diploma per Tammugae, connesso al paleosardo Taтmисаr, e per Bonossonis, legato al toponimo Bonossai): le rilevate congruenze con i nomi libici, piuttosto che riportarsi alla relegazione di popolazione libica in Sardegna ad opera di Cartagine, nel quadro della politica cerealicola della capitale punica, sarebbero da ascriversi alla componente libica del paleosardo prefenicia (PAULIS 1987, XXVII) in connessione con la diffusione di toponimi di radici affini diffusi nelle aree interne dell'isola, in cui l'elemento culturale punico è fortemente minoritario. Si noti in particolare il dat. Iurini e il dat. Tisareni, rispettivamente dell'uxor e di una filia di Hannibal, affini al dat. femm. Nispenini, analizzato da L. Gasperini come nome paleosardo in trascrizione latina da Olbia, e al dat. maschile Nercauni con un nominativo Nercau (GASPERINI 1996, 311-316). L'origo di Hannibal ci riporta con certezza ad un centro demico di origine protosarda denominato con un sintagma paleosardo-latino $\operatorname{Nur}(a x)$ alb(us), sorto presso un nuraghe caratterizzato dal materiale costruttivo bianco. Su tale processo denominativo di centri di età romana, sopravvissuti nel medioevo, ci soccorre un Nuraci Albu, villa medievale, presso Oristano-Il Rimedio, attestato nel 1388 come Nuraci Albu e successivamente come Nuracraba (da *Nuracalba $>$ *Nuraclaba $>$ Nuracraba, per un incrocio paretimologico con craba-capra), estinto nel 1652 (ToLA 1861, 843 con errata trascrizione Nurau albu. Cfr. CASUla 2006, 2320-2321, 2329).

49 CIL X 8004. Cfr. Atzori 2006, 125-127, nr. 3: ++(M.P.) / [Im]p(erator) Caes[ar di]vi Nerv[ae f(ilius)] / [N]erva Tr[aianus] Augus[tus] / Germ(anicus) [Dac(icus)] Pontif[ex M]a[x(imus)] / [tr]i[b(unicia)] pot(estate) [- - - im]p(erator) VI, C[o(n)s(ul) - - ] / [- - ]++++[- - ]++ / d[u]cit ve[tusta]te corrupta[m] / [C.] Vlpi[o Se]v[ero proc(uratore) Aug(usti)] / [praef(ecto) pro]vi[nciae Sard(iniae)] / [restituit] (106 d.C.).

50 Thomasson 2009, 6, nr. 2:050: "C. Ulpius Severus. - Haud ante Traianum. - proc. Aug. praef. prov. S[ard.] NSA 5 (1929) 319-323 = Sotgiu, Iscr. I $221=A N R W$ II: 11/1, p. 569 (A 221) $=A E$ 1990, 451 = L'Africa romana 9 (1991) 574-576 = AE 1992: 891. - Meloni 195 sq., n. 16. Cf. etiam A. E. Astin, Latomus 18 (1959) 151, et quae 'Zur Verwaltungsgeschichte der Provinz Sardinia', Eranos 70 (1972) 72-81, disserui'.

51 ILSard I $221=A E$ 1990, $451=A E$ 1992, 891. Fonni: [Di]ana[e et] Silvano / [n]emoris Sorabensis / C(aius) Ulpius Severus / proc(urator) Aug(usti) / praef(ectus) prov(inciae) S[ar(diniae)]. 
Se questa ipotesi cogliesse nel segno potremmo attribuire a Lucio Cossonio Gallo una serie di atti che parrebbero connessi fra di loro. Innanzitutto il proconsole potrebbe essere stato l'effettivo curatore della riduzione della Sardinia, amministrata dal Senato, a provincia inermis. La Sardegna che, nel periodo più acuto del bellum che la sconvolse a partire dal 6 d.C., aveva annoverato fino a cinque cohortes auxiliariae e per oltre un decennio (19-31 d.C.) il corrispettivo di una legione, composto da liberti di religione giudaica o alessandrina, era ancora nel 102 dotata delle due cohortes geminae I Sardorum et Corsorum e II Gemina Ligurum et Corsorum.

Con grande probabilità la prima cohors gemina fu sciolta e al suo posto fu rifondata la cohors I Sardorum, forse dotata dell'epiteto praetoria, mentre la cohors II Gemina Ligurum et Corsorum fu trasferita in Oriente, presumibilmente in previsione delle campagne traianee contro l'Armenia e la Partia e per sedare il tumultus iudaicus.

\section{La constitutio del Forum Traiani in Sardinia}

A celebrare la conclusione del secolare conflitto fra le civitates Barbariae e l'area romanizzata ad occidente del plesso montano centrale, il proconsole poté dare attuazione alla volontà di Traiano della fondazione di un nuovo centro, Forum Traiani, presso le Aquae Ypsitanae di Fordongianus, che avevano ospitato la cohors I Corsorum e presso le quali, sotto Tiberio, alcune (o le universae) civitates Barbariae avevano posto una targa ad un edificio in onore dell'imperatore. Questa nuova fondazione era connessa, inoltre, all'unificazione dei due tronconi viari a Turre e a Karalis nella via a Karalibus Turrem, ottenuta con la costruzione del ponte sul Thyrsus fluvius presso le Aquae Ypsitanae e con la costruzione della via da Aquae Ypsitanae ad Aquae Neapolitanae, attraverso Othoca, con la contemporanea edificazione del ponte sul Rio Palmas, a sud di Othoca. ${ }^{52}$

La constitutio di Forum Traiani, ossia di un centro -forum-negotiationis locus ut f(orum) Flaminium, f(orum) Iulium ab eorum nominibus qui ea fora constituenda curarunt ${ }^{53}$ si ascrive, sulla base del suo nome al principato di Traiano (98-117 d.C.), benché le fonti, proprio di età traianea, della nesos Sardo di Tolomeo registrino esclusivamente gli Hydata Hypsitana, probabilmente perché la constitutio avvenne nella fase tardiva del principiato traianeo.

Il recente filone di studi sui vici, fora e conciliabula ${ }^{54}$ ha portato a conclusioni di estremo interesse anche per il caso del Forum Traiani della Sardinia.

L'integrazione della lacuna iniziale del lemma vicus nel compendio di Festo del De verborum significatu di Verrio Flacco consente di intendere i tre modus dei vici. A prescindere dal vicus come tipo di edificio dove si ricoveravano i lavoratori dei campi nelle regioni prive di villa, le due altre tipologie di vici si dividono da un lato

52 Sulle opere viarie e, in particolare, sulla costruzione e restauro di ponti, in Italia e nelle provinciae, esplicitamente ricordati da Dio LXVIII, 15, 3 cfr. UGGERI 1983, 232-236; Migliorati 2003, 113-114.

53 Festvs, p. 84, ed. Mueller.

54 SisANi 2011, 547-780, con bibl. precedente, e Todisco 2013. 
perché habent rempublicam et ius dicitur, dall'altro nihil eorumet tamen ibi nundinae aguntur negoti gerendi causa, et magistri vivi, item magistri pagi quotannis fiunt.

Nella pregnante traduzione di Simone Sisani il passo suona così:

Ma tra i vici alcuni sono dotati di res publica e vi si amministra la giustizia, altri non hanno nulla di tutto ciò e tuttavia vi si tengono le nundinae ai fini del commercio, ed ogni anno vi si eleggono sia i magistri vici, sia i magistri pagi. ${ }^{55}$

In definitiva la distinzione fra vici verte sulla iurisdictio e sulla dotazione di una res publica, presenti nei vici del primo tipo, ed assente in quelli di secondo tipo, che dispongono dei magistri vici, privi di iuriusdictio, che compete alla colonia o al municipium entro il cui territorium sono incardinati i vici, mentre le nundinae sono appannaggio di entrambe le tipologie di vicus. Applicando la distinzione al caso in esame potremmo dire che le Aquae Ypsitanae furono probabilmente un vicus del secondo tipo, con le nundinae, forse i magistri vici o magistri pagi, dipendenti dalla colonia iulia augusta Vselis, mentre il Forum Traiani fu un vicus del primo tipo, dunque una comunità non urbana, ma rurale, dotata di res publica e di iurisdictio autonoma. Secondo la brillante proposta di Simone Sisani, sostenuta da Filippo Coarelli, Silvio Panciera, Luigi Capogrossi Colognesi e Umberto Laffi, nei vici di primo tipo, con respublica e iurisdictio, devono riconoscersi due categorie di comunità, documentate da leges e da testi epigrafici e letterari, i fora e i conciliabula. Se questi ultimi erano prevalentemente incentrati sui santuari, i fora rispondevano ad un'altra logica: si tratta di centri di ambito vicano che, comunque, si richiamano ad una organizzazione in nuce urbana. ${ }^{56}$

L'epoca di constitutiones dei fora è essenzialmente quella dell'estrema media repubblica e tardo repubblicana.

In età cesariana assistiamo alla formazione di vari Fora Iulii. Sotto il principato le constitutiones di fora diminuiscono drasticamente: a fronte di alcuni casi di Forum Augusti (nella penisola iberica, forse in Sardinia, Austis), si ha un solo Forum Tiberii, alcuni Fora Claudi (soprattutto in ambito alpino e provinciale), un unico Forum Neronis.

Nel II secolo abbiamo le due ultime constitutiones di fora: il Forum Traiani in Sardinia e il Forum Hadriani in Britannia. Per il caso sardo, come ha recentemente notato Cira Crespo Cabillo, dovremmo avere uno stabilirsi di coloni (forse, nell'ipotesi di Marc Mayer, ${ }^{57}$ veterani delle campagne daciche) che ricevevano individualmente lotti di terra, con la formula dell'adsignatio viritim. ${ }^{58}$

\footnotetext{
55 SiSANi 2011, 559.

56 SISANI 2011, 543-545, 593-594.

57 Marc Mayer viva voce.

58 CRespo CABillo 2009, 289.
} 


\section{Topografia di Forum Traiani}

Il Forum Traiani rappresenta nell'ambito della poleografia della Sardinia interna un caso unico: si tratta infatti di un centro di fondazione che si giustappose ad un agglomerato termale-militare preesistente. ${ }^{59}$

Pur nella limitata estensione della fondazione traianea parrebbe chiaro che anche nel Forum Traiani della Sardinia si palesi quella "«militarisation» de l'urbanisme trajanien" su cui si è soffermato recentemente Pierre Gros, analizzando il foro di Traiano in Roma. ${ }^{60}$

L'area prescelta per la fondazione del Forum è costituita da una bancata trachitica livellata (quote fra 38 e $37 \mathrm{slm}$ ) presumibilmente priva di un agglomerato preesistente, in quanto le Aquae risultano collocate sulla riva sinistra del fiume Tirso, a quote fra i m 35 e i $25 \mathrm{slm}$, al disotto della bancata di trachiti, mentre i castra, se correlati all'anfiteatro, sarebbero stati allocati a circa 750 metri a sud dell'area del Forum Traiani.

Il centro di fondazione, forse legato ad un trapianto di veterani traianei, appare costituito con un impianto rigorosamente quadrangolare, con strade che si intersecano ad angolo retto.

Il Forum Traiani corrispondeva al punto mediano della nuova viabilità centrale della Sardinia, la via a Turre Karales, che raccordava l'antica via a Turre con il nuovo tracciato della via per Karales attraverso Othoca ed Aquae Neapolitanae, mediante un ponte ${ }^{61}$ a sette arcate (oggi scomparse) impostate su sei piloni in cementizio, rivestiti in opera quadrata in trachite grigia, a profilo leggermente convesso a valle, mentre a monte dotati di rostro acuto.

Il ponte (Fig. 1, 1), presumibilmente traianeo, ${ }^{62}$ costituisce l'asse generatore, con orientamento NNO / SSO del Forum, sul cui prolungamento in direzione SSO si disponeva il cardo I. Tale cardo non corrisponde, nonostante le apparenze, alla via Ipsitani, aperta nel tardo Ottocento, bensì alla linea divisoria dei mappali 433 e 434 di fondi rustici, attigui all'abitato, del catasto urbano del 1909, redatto dal geometra Cipriani (Fig. 1, 2). Tale linea è normale, nel medesimo catasto, al divisorio fra $\mathrm{i}$ mapp. 433, 630, 432, 431, 429 e 449, 448, 447, 446, 437, probabilmente erede del decumanus meridionale (Fig. 1, 3).

Il parcellario catastale testimonierebbe così gli assi stradali estremi NNO / SSO e ENE / OSO della fondazione traianea.

In occasione di una verifica sul terreno, in data 20 marzo 2008, si è documentato lungo il primo limite fra i mappali 433 e 434, una lunga sequenza di basoli in basalto (dimensioni massime: $\mathrm{m} 1$ x 0,60), consunti dall'uso da parte di veicoli, con tracce evidenti di carraie, riutilizzati come elementi del muro di macera, ma pertinenti con tutta evidenza al basolato del cardo I est. Nella stessa area si rilevano numerosi

59 ZuCCA 2009, 573-586.

60 Gros 2000, 227-249. Si vedano in particolare gli esempi "classici" delle fondazioni "militari" di Thamugadi e Cuicul, ma soprattutto nella prima: cfr. CAMPos CARrasCo - Pérez Macías 2003, 269-300.

61 Lunghezza m 120; largh. m 5. Cfr. Galliazzo 1995, 170, nr. 360.

62 Galliazzo 1995, 170, nr. 360, con riferimento al ponte di Alconétar (Spagna) ugualmente traianeo. 


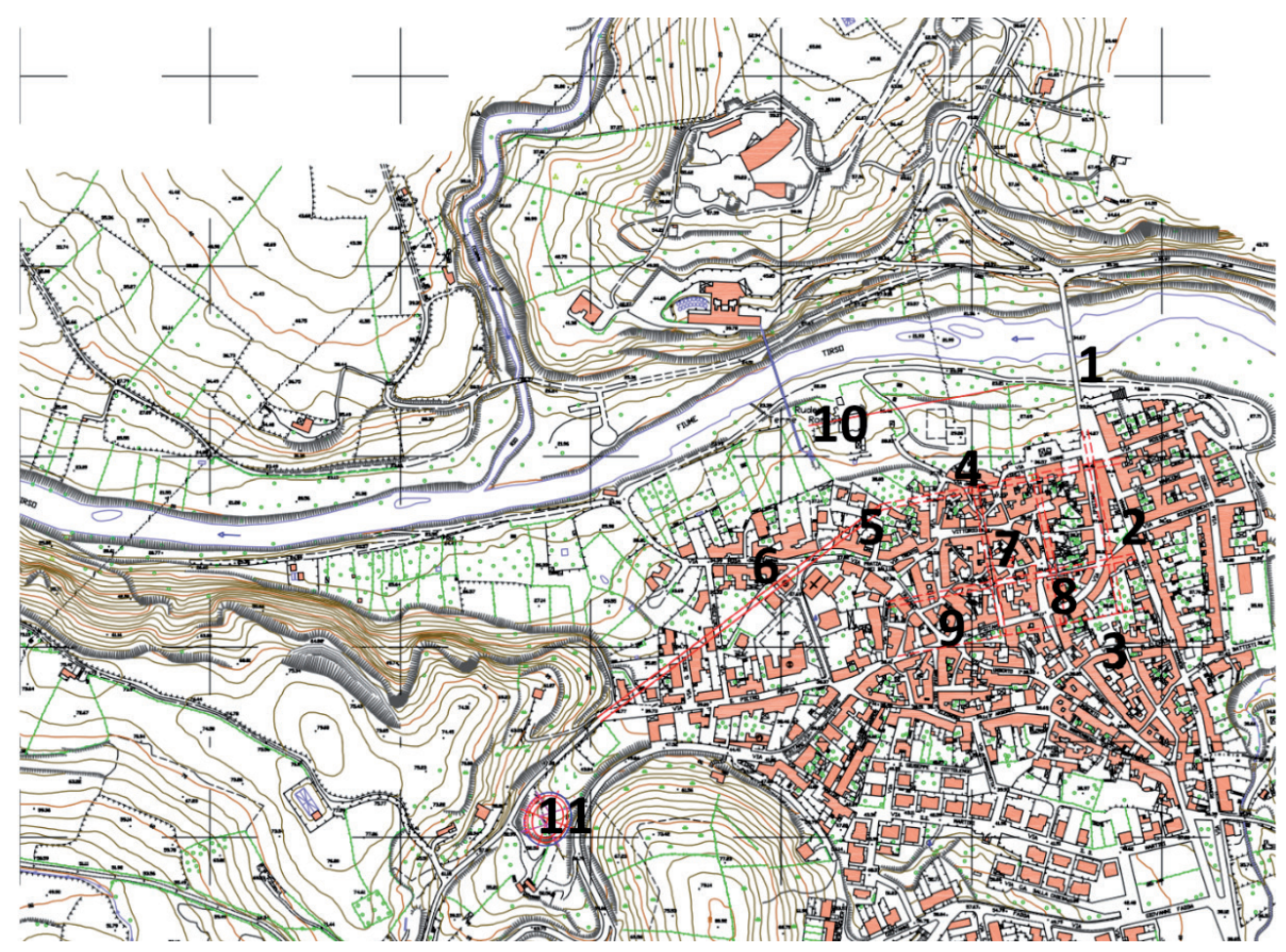

Fig. 1. Particolare del piano aerofotogrammetrico del Comune di Fordongianus, rielaborato da Tore Ganga. Legenda: 1.- Ponte traianeo sul Thyrsus fluvius. 2.- Cardo I. 3.- Decumanus meridionale. 4.- Decumanus settentrionale. 5-6.- Via nova per l' anfiteatro diretta a Karales. 7.- Terme centrali. 8.- Edificio con volta a botte. 9.- Edificio industriale. 10.Aquae Ypsitanae. 11.- Anfiteatro.

blocchi squadrati in trachite ed embrici di pasta rosso-arancio, pertinenti a strutture romane.

Il reticolo viario regolare del Forum Traiani si desume anche da un tratto stradale urbano, orientato ENE / OSO, messo in luce il 4 novembre 2002, durante lavori edilizi in Via Vittorio Veneto 45 (proprietà Dante Nughes). La via, larga m 5,40 (18 pedes), conservata per una lunghezza di $\mathrm{m} 7$, dotata di crepidines, in conci di basalto, lunghi $\mathrm{m} 0,59$, larghi $\mathrm{m} 0,25$, alti $\mathrm{cm} 30$, presenta il summum dorsum bombato, basolato con lastre di basalto di grandi e medie dimensioni (dimensioni massime $\mathrm{cm}$ 90 x 40). Si tratterebbe del decumanus I settentrionale della fondazione traianea (Fig. 1, 4).

Dall'estremità occidentale di questo decumanus si dipartiva un asse viario orientato NE / SO, diretto verso l'anfiteatro, evidenziato nel corso dei lavori di demolizione di uno stabile prospicente il Municipio, nel maggio 1993. La strada, messa in luce (e distrutta) per un tratto di $\mathrm{m} 12$, era lastricata con basoli poligonali in trachite grigia 
(dimensioni di tre basoli: $\mathrm{cm} 120$ x 90; $\mathrm{cm} 101$ x 87; cm 70 x 53), larga m 5,37, con crepidines laterali costituite da blocchi ben sagomati di trachite di $\mathrm{cm} 29$ x 29 x 22 di altezza $^{63}$ (Fig. 1, 5).

Infine dirimpetto al vecchio municipio, lungo la via Traiano, fu individuato il 7 maggio 1969 da parte del funzionario della Soprintendenza alle Antichità di Cagliari Luigi Frongia un ulteriore tratto stradale, con il condotto fognario centrale di $\mathrm{m} 0,60$ di larghezza e di $\mathrm{m}$ 0,45 di profondità, da ritenersi diretto, con il medesimo orientamento NE / SO, verso l'anfiteatro (Fig. 1, 6).

Ad assicurarci dell'orientamento del reticolo viario, e di conseguenza delle insulae dell'abitato, lungo gli assi NNO / SSO e ENE / OSO, sono i resti di tre complessi edilizi, ancora oggi rilevabili, che presentano le murature perimetrali orientate secondo gli assi suddetti.

1)Terme centrali (Fig. 1, 7)

Edificio individuato nel 1952 e nel maggio 1969 nell'area di Vico Doria (civici 11-13-15), Via Dante e Via Vittorio Veneto 11.

Della struttura si è rilevato un ambiente caldo rettangolare, orientato ENE / OSO, di $\mathrm{m}$ 3,70 residui di lunghezza x 6,40 di larghezza, forse un tepidarium, in opera cementizia con paramento in opus vittatum di tufelli, ${ }^{64}$ con impiantito di bessales su cui si impostavano le suspensurae di pilastrini litici di $\mathrm{cm} 20$ × 20 × 60 di altezza, che reggevano un pavimento sospeso formato da bipedales ( $\mathrm{cm} \mathrm{58,2}$ x 59,1 x 7). Il lato breve ENE comunicava con un vano di circa $30 \mathrm{mq}$, in opera cementizia rivestito in opus vittatum mixtum, orientato con i lati brevi in direzione NNO / SSE e corrispondente ai cortili dei civici 13 e 15 di Vico Doria e, parzialmente, allo stesso Vico Doria. L'ambiente presentava un pavimento musivo di m 9,15 x 2,98 / 3,40 / 1,76 di dimensioni residue. Il mosaico, trasferito al Museo Archeologico Nazionale di Cagliari è stato studiato da Simonetta Angiolillo nel suo Corpus dei mosaici antichi della Sardinia: "Il campo è delimitato da un bordo ... decorato a dallage... Lo schema compositivo del campo è basato sull' alternanza di quadrati, sui cui lati si impostano pelte, e di cerchi ... Il motivo ampiamente documentato in Africa ritorna in Sardegna a Tharros nel c.d. Tempio a pianta di tipo semitico". Per il nostro esemplare l'Angiolillo si è riferita in particolare ad un pavimento della Casa delle fatiche di Ercole di Volubilis, in Mauretania Tingitana, della fine del II-inizi del III sec. d.C., coevo a questo di Forum Traiani.$^{65}$ L'ambiente in questione deve identificarsi, con grande probabilità, con il frigidarium delle terme. Presumibilmente allo stesso

63 Il basolato stradale giaceva a $\mathrm{m} \mathrm{1,08} \mathrm{di} \mathrm{profondità} \mathrm{rispetto} \mathrm{al} \mathrm{marciapiede.} \mathrm{Nell'interro} \mathrm{del} \mathrm{piano}$ stradale, costituito da uno strato di terra marroncina con ciottoli e scarso vasellame comune si è individuato un frammento di sigillata chiara D 2 (bizantino).

64 Tufelli di $\mathrm{cm} 29$ x 9; strato di malta regolare di $\mathrm{cm} \mathrm{2,5.} \mathrm{Il} \mathrm{paramento} \mathrm{era} \mathrm{rivestito} \mathrm{di} \mathrm{due} \mathrm{strati} \mathrm{successivi}$ di intonaco, per uno spessore complessivo di $\mathrm{cm} 3$.

65 Angiolillo 1981, 155. Appare incerto stabilire l'identificazione del mosaico in questione con il mosaico individuato nell'Ottocento, in un "tratto di via" di Fordongianus: "In Fordongianus un tal Antonio Spano, scavando in un tratto di via dentro lo stesso villaggio alla profondità d'un metro e più, trovò un pavimento ornato di mosaico lavorato a diversi colori molto bello ed intiero. Rappresenta un ornato di foglie e di pampini che fanno un bel risalto in giro, ed in mezzo avvi una gran ghirlanda ben intrecciata, con altre quattro più piccole agli angoli dello stesso mosaico" (SPANo 1874, 22). 
edificio termale corrisponde il tratto murario in opus vittatum mixtum, di m 3,80 di lunghezza, conservato in altezza per m 1,1, tra via Dante e Via Vittorio Veneto.

2) Edificio con volta a botte (Fig. 1, 8)

L'edificio, a pianta rettangolare, orientato ENE / OSO, insiste nelle attuali proprietà di Mariano Spano e Archelao Zedda, corrispondenti agli immobili attigui di via Vittorio Veneto 6, 8, 10.

La struttura in cementizio con paramenti in opus vittatum mixtum, si estende in lunghezza per $\mathrm{m} 22 \mathrm{e}$ in larghezza per $\mathrm{m}$ 10. L'altezza delle murature è attualmente di $\mathrm{m}$ 2,30 dal piano di calpestio, sopraelevato, secondo fonti orali, rispetto al pavimento della struttura romana di $\mathrm{m} 2,20$. Sul lato lungo NNO è visibile l'imposta della volta a botte, in opera cementizia, articolata in quattro ricorsi di laterizi che dovevano probabilmente formare una rete a linee parallele, sistema divulgato da Traiano in poi, in specie nella seconda metà del II sec. d.C. Il modulo dell'opus vittatum mixtum corrisponde a quello delle Terme II, a riscaldamento artificiale delle Aquae Ypsitanae.

Nella proprietà Spano si individua il prospetto dell'edificio, normale al lato lungo, realizzato in cementizio con paramento in opus vittatum mixtum, intonacato, spesso $\mathrm{m} 0,86$, dotato di una apertura, di dimensioni non definite, a $\mathrm{m} 0,45$ dall' angolo fra $\mathrm{i}$ due muri. Il primo vano accessibile dall' apertura ha una lunghezza di 10 pedes (m

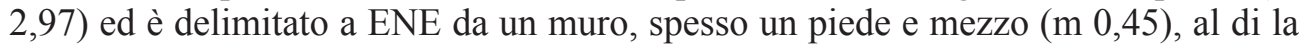
del quale, nella proprietà di Archelao Zedda, si estende la parte restante dell'edificio, delimitato sul lato lungo SSE dal muro in cementizio, con rivestimento esterno ed interno in opus vittatum mixtum. Ignoriamo la funzione della struttura, che parrebbe di carattere pubblico.

Secondo il proprietario Mariano Spano la struttura romana sarebbe stata riadattata ad edificio chiesastico nel medioevo, ${ }^{66}$ in funzione di un monastero dell'ordine di San Giacomo di Altopascio, cui si riferirebbe un sigillo in bronzo, rinvenuto presso il ponte romano di Forum Traiani, tra il 1780 e il 1795, di un frater Mart(inus) m(a) g(iste)r Hosp(italis) d(e) S(an)c(to) Iac(opo) d(e) Alt(o)pa(sso), il maestro dell'Ordine fra il 1108 e il $1146 .{ }^{67}$

3) Edificio industriale (Fig. 1,9)

All'interno dello scantinato dell'abitazione di Pietro Zedda, ubicata fra Via Ipsitani 113-115 e via Vittorio Veneto, si individua un ambiente rettangolare, ridotto a due spezzoni di muro, orientati rispettivamente SSE / NNO (per una lunghezza residua di $\mathrm{m} 2,19$ ) e ONO /ESE (per una lunghezza residua di $\mathrm{m} 3,33$ ). Su questo ultimo lato, addossate alla muratura, erano disposte due vasche rettangolari, ad angoli interni stondati), dotate di foro di scarico affinché il liquido contenuto nella prima vasca fluisse, depurato, nella seconda vasca.

La struttura muraria è in opus vittatum, in filari regolari di tufelli in trachite $(\mathrm{cm}$ 18 / 20 / 21 di lunghezza x cm 7 di altezza), connessi con strati di malta di $\mathrm{cm} 2$ di spessore.

66 ZEDDA 1906, 61-62, parrebbe alludere a questa struttura, identificata come chiesa di un monastero Vallombrosano, non altrimenti attestato.

67 BAILLE 1797. 
Dall'area archeologica provengono tegulae hamatae, forse connesse alla deumidificazione di ambienti, lastrine in marmo bianco (spessore $\mathrm{cm} \mathrm{14),} \mathrm{embrici} \mathrm{giallastri}$ e rossastri e ceramica comune romana (bocca di un askòs, ceramica "fiammata" di bottega sarda, sulcitana?), lucerne a becco tondo, anfore Africane e un asse di Adriano del 134/138 d.C. ${ }^{68}$

L'edificio parrebbe avere avuto una fase di laboratorio industriale per la torchiatura delle olive o per la produzione vinaria.

Non possediamo allo stato delle conoscenze dati per l'individuazione della piazza forense di Forum Traiani.

Una serie di iscrizioni marmoree relative ad interventi evergetici o a onoranze ad imperatori potrebbero riferirsi all'area forense, ma è dubbia la circostanza puntuale del loro riferimento.

Il 25 maggio 1928, in Via Traiano, dirimpetto alla chiesa parrocchiale, vicino alla casa di Giuseppe Uselli, ${ }^{69}$ durante i lavori per la posa di tubi dell'acquedotto si individuò "una pavimentazione a smalto di calcestruzzo e una piccola tubazione [forse il fondo di una vasca o meglio di una cisterna]" e "parecchie monete di poco valore"70 insieme a un frammento di lastra di marmo bianco con venature grigie recante una iscrizione: ${ }^{71}$

$$
\text { - - - - - / [- - ]VER [- - / - - ex] test(amento) [- - / D(- - -)] D(- - - })^{72}
$$

Il testo, richiamante un intervento (evergetico?) [ex] test (amento), all'ultima linea presenta, centrato, un interpunto triangolare che impone la probabile integrazione di una seconda $D$, di cui parrebbe cogliersi in frattura la curva esterna. Le soluzioni della sigla $D$. $D$. sono naturalmente molteplici, ma la monumentalità del testo induce a non escludere lo scioglimento d(ecurionum) d(ecreto).

Dall'area di Via Bagni, corrispondente probabilmente a via delle Terme, poiché il primo editore Ettore Pais specifica "nel centro del paese" 73 provengono frammenti marmorei pertinenti a tre distinte iscrizioni imperatorie, da supporsi affisse nel forum o nell' augusteum di Forum Traiani.

68 RIC, II, Hadrian, 466, nr. 841.

69 Archivio Soprintendenza Archeologica di Cagliari e Oristano. Fordongianus. Lettera di A. Zedda a A. Taramelli del 25 maggio 1928.

70 Archivio Soprintendenza Archeologica di Cagliari e Oristano. Fordongianus. Lettera di A. Oppo Palmas a A. Taramelli del 27 maggio 1928.

71 Alt. cm 38; largh. cm 21; spess. cm 3; alt. lettere $\mathrm{cm} 7$.

72 ILSard I 201 (Cagliari, Museo Archeologico Nazionale).

73 PAIS 1894, 921. 
1) Dedica a Caracalla (4 febbraio 211-213 d.C .) ${ }^{74}$

- - - - - pio / [fel(ici)] Aug(usto) pon[t(ifici) / m]ax(imo), trib(unicia) po/ [testate) - - -]I, co(n)s(uli) III, p(atri) p(atriae) / [proco(n)]s(uli), divi Severi / [fil(io), - - - ]+++/ - - - - - 75

2) Dedica a Severo Alessandro (222-235 d.C. $)^{76}$ [Imp(eratori] Cae[s(ari) / M. Aur]elio S[evero / Alexandro, p]i[o fel(ici) / - - - - -

3) Dedica ad un imperatore Dominus noster (? $)^{77}$
a) $D$
b) [- - f]ilio[- - - ]
c) - - - - - / ++++N[- - - / Dom[ino nostro? - - -/ - - - ]++/

Si aggiungano due dediche ad imperatori anonimi pro salute rinvenute dell'area urbana e connesse al forum o all' Augusteum della città. ${ }^{78}$

L'importanza di Forum Traiani si palesa anche nella monumentalizzazione delle precedenti Aquae Ypsitanae ${ }^{79}$ (Fig. 1, 10) e nell'anfiteatro (Fig. 1, 11), forse di origine castrense di età augustea e ampliato in periodo severiano. ${ }^{80}$

L'orientamento del complesso termale è il medesimo del Forum Traiani, così da autorizzare l'ipotesi di una programmazione generale urbanologico delle Terme e del Forum ad opera di Traiano, ovvero la strutturazione del Forum secondo gli assi delle Aquae Ypsitanae.

\section{La carriera di L(ucius) Cossonius Gallus dopo il proconsolato della provincia Sardinia}

Il proconsole Lucio Cossonio Gallo dovette gestire il governo della Sardinia probabilmente per un solo anno, poiché il senatore rivestì in progresso di tempo due ulteriori incarichi prima di giungere al consolato suffectus nel 116 d.C.

Dalla Sardinia Cossonio ritornò a Roma dove gestì il rango sacerdotale di sodalis Flavialis, probabilmente nel 112 d.C. ${ }^{81}$

74 ILSard I 189 = ELSard A 189, p. 568. Tre frammenti di lastra di marmo recuperati dal sindaco di Fordongianus Antonio Oppo anteriormente al 1894. Fino al 1960 era presente nella collezione Oppo-Palmas di Fordongianus il frammento sinistro, ora disperso insieme agli altri. Dimensioni del framm. sin. alt. cm 24; largh. cm 15; spess. cm 1,5; alt. lettere $\mathrm{cm} 6$.

75 Per la posposizione del patronimico imperiale all'indicazione del consolato e del proconsolato, meno comune rispetto alla anteposizione di seguito ad Imp. Caes.; cfr. ad es. CIL VIII $4197=I L S 450$.

76 ILSard I 190 = ELSard A 190, p. 568. Il frammento di lastra marmorea fu recuperato nelle medesime circostanze dell'iscrizione precedente, cui il frammento in esame non apparterrebbe per le differenze dimensionali e di ductus rilevate dal primo editore, Ettore Pais.

77 ILSard I $200=$ ELSard A 200, p. 568. I tre frammenti di lastra marmorea con una iscrizione imperatoria, furono rinvenuti nelle stesse condizioni di giacitura dei nrr. 1-3. Sino al 1960 erano conservati nella collezione Oppo-Palmas due dei tre frammenti, attualmente dispersi.

78 ZuCCA 1994, 914, note 331-3.

79 Gніотто 2004, 109-111; 131-132.

80 BACCo et alii 2010, 1371-1460.

81 RÜPKE - GLOCK 2005, 930-931, nr. 1404. 
Fra il 113 e il $115^{82}$ Cossonio ebbe il governo-leg(atus) Aug(usti) pro pr(aetore)nelle provinciae imperiali di Galatia ${ }^{83}$ Pisid(ia $)^{84}$ e [P] aphlagonia, ${ }^{85}$ probabilmente in occasione della riforma provinciale di Traiano, nell'Asia Minore, che stabilì la separazione fra la provincia Cappadocia e quella di Galatia. ${ }^{86}$ Infatti la nuova provincia della Cappadocia è retta dal legatus consolare M. Iunius Homullus (111/112113/114) ${ }^{87}$ e successivamente, nel 114-117, da L. Catilius Severus Iulianus Claudius Reginus $^{88}$ legatus consolare della Cappadocia, dell'Armenia e dell'Armenia Minor. ${ }^{89}$

La scelta di Lucio Cossonio come legato della Galatia parrebbe legata alla volontà di Traiano di disporre di suoi uomini, esperti in amministrazione provinciale e in responsabilità militari, nel cruciale momento delle campagne contro l'Armenia e, successivamente, la Partia.

Il 27 ottobre $113^{90}$ Traiano lasciò Roma imbarcandosi, probabilmente a Brundisium, sulla quadriere Ops, comandata dal praefectus classis Misenatis Q. Marcius Turbo ${ }^{91}$ e dirigendosi a Corinto e ad Atene attraverso il periplo del Peloponneso. Ripreso il viaggio da Atene la quadriere Ops con l'Imperatore si diresse al caput provinciae Asiae Ephesus. Da qui l'imperatore proseguì via Aphrodisia fino a Pattara o Attaleia, dove si reimbarcò sull' Ops, che tenne una rotta costiera attraverso la Cilicia fino a Seleucia di Pieria e da qui ad Antiochia, dove Traiano giunse il 7 gennaio $114 .{ }^{92}$

Si è insistito sulla descrizione di questo viaggio poiché tra i milites della quadriere Ops vi era un anonimo sardo che, ricevette l'honesta missio il 114 da Traiano, come attesta il diploma militare rinvenuto a Olbia (ant. Terranova) e ceduto al Metropolitan Museum of Art di New York. ${ }^{93}$ Secondo Yann Le Bohec solamente "le lieu de

82 ECK 2007, 205; ID. 2013. La cronologia del governo della Galazia da parte di Cossonio è estremamente discussa, tuttavia l'acquisizione del dato del consolato suffectus al 116 d.C. e non al 119 come ipotizzato in precedenza rende le datazioni basse, post 115 d.C., non più sostenibili: SHERK 1951, 63-65; ID. 1979, 168, nr. 3 (117-119 d.C.); Thomasson 2009, 103, nr. 28:019 (ultimi anni di Traiano); RüPKE - GlocK 2005, 930 (113-115 d.C.); Urloiu 2010, 3 (117-119 d.C.); Hillebrand 2006, 251, nr. 15 (119 d.C.).

83 RÉmY 1986; ID. 1989; STROBEL 1998.

84 BRANDT 1992.

85 MAREK 1993; ID. 2013.

86 L'evento è riferito al 113 d.C, da Strobel 2013b. Su altre cronologie, all'interno degli ultimi anni del principato traianeo cfr.VAgLieri 1910, 361-3 (100 d.C.); Luzzatto 1985, 309 (107 d.C.). Sull'origine della provincia romana della Galatia cfr. Mitchell 1993, 73-91; SHERK 1979, 166-175; ID. 1980, $954-960$. Sull'apporto italico alla popolazione della provincia (che giustifica il titulus onorario in latino di Cossonio) cfr. VALVO 2007, 151-163.

87 PIR ${ }^{2} \mathrm{~J} 760$.

88 ECK 2013.

89 Teja 1980, 1083-1124; STrobel 2013a.

90 Fonti e bibliografia su questa data in Migliorati 2003, 132.

$91 P^{2}, \mathrm{~V} / 2,1983,188-190$ (M 249).

92 Guey 1937, 43-44; Lepper 1948, 28-30; Migliorati 2003, 133-134. Incerto sul ruolo della quadriere Ops e di Q. Marcio Turbone nel viaggio di Traiano in Oriente del 113-114 è BérARD 1984, 294, n. $104,306$.

93 CIL XVI 60 (p. 215) = ILSard I $311=$ AE 1927, 3. Terranova / Olbia: Imp(erator) Caesar [Divi Nervae f(ilius) Nerva] / Traianus O[ptimus Aug(ustus) Germ(anicus)] / Dacic(us), pon[tif(ex) maxim(us), tribu]/nic(ia) potesta [te XVIII], / imp(erator) $\overline{V I}$, [co(n)s(ul) VI, p(ater) p(atriae)], / iis qui naviga[verunt in qua]/driere Ope et [militaverunt in] / classe praetor[ia Misenensi] / sub Q(uinto) Marcio Tu[rbone quorum] / nomina subsc[ripta sunt ipsis] / liberị[s posterisque eorum civitatem dedit],// Imp(erator) Caesar Divi N[ervae f(ilius) Nerva] / Traianus Optimu[s Aug(ustus) Germ(anicus) Da]/cic(us), pontif(ex) ma[xim(us), tribunic(ia) p]/ọtestat(e) X[VIII, imp(erator)] $\overline{V I}, \operatorname{co}(n)[s(u l) V I, p($ ater $)$ p(atriae)] / [- - - ]+[- - -]. 
la découverte [Olbia] établit un lien avec la Sardaigne", ${ }^{94}$ tuttavia è rilevante una presenza fra i classiarii della Ops di un sardo, privo della cittadinanza romana, che probabilmente prese parte alle prime fasi della campagna orientale di Traiano. ${ }^{95}$

Ritornando al legatus augusti propraetore della Galatia Lucius Cossonius Gallus, egli ebbe certamente un ruolo organizzativo delle truppe per la campagna contro l'Armenia, in quanto sappiamo che il suo caput provinciae Ancyra ospitò, durante la sua legazione, fra il 113 e il 114, le truppe danubiane destinate ad unirsi alle armate orientali di Traiano, ${ }^{96}$ che vennero nutrite a spese del senatore C. Iulius Severus, discendente dai dinasti galati. ${ }^{97}$

Lucio Cossonio fu onorato con una statua recante il suo cursus honorum ad Antiochia di Pisidia ${ }^{98}$ così come la sua uxor Clodia Iatrina, ${ }^{99}$ ricevette una onoranza a Iconium a cura della figlia di C. Eburenus Maximus, archiereus del culto imperiale. ${ }^{100}$ Cossonio lasciò la Galatia nel 115 per ritornare a Roma dove rivestì il sacerdozio dei septemviri epulonum, evidentemente poiché si era reso libero uno dei dieci posti di tale sacerdozio, prima o dopo essere consul suffectus nel 116. ${ }^{101}$

L'ulteriore incarico fu quello di legatus consolare della Iudaea intorno al 120, rivelato da una base di statua frammentaria con iscrizione onoraria di Caesarea Maritima. ${ }^{102}$

Probabilmente fra le cospicue milizie della Iudaea Cossonio ritrovò anche, tra le forze ausiliarie, la cohors II gemina Ligurum et Corsorum che, con verosimiglianza, egli aveva avviato dalla Sardinia in un teatro militare orientale.

\footnotetext{
94 LE BOHEC 1990, 120, nr. 37.

95 Mastino 2009, 145.

96 Sui problemi degli itinerari seguiti da Traiano da Antiochia per l'invasione dell'Armenia, cfr. ANGELI BerTinelLi 2000, 28-29, n. 10.

97 IGGR, 3, 173; SEG XVII, 142; French 2003, 105, nr. 14; Mitchell - French 2012, 20-22, 27-31, nrr. 72-76. Cfr. Migliorati 2003, 132, 140-141, per l'itinerario seguito da Traiano dalla Siria al confine orientale della Cappadocia e BARREsi 2003, 567, per il ruolo ad Ancyra rivestito nel frangente della concentrazione delle truppe danubiane in vista del ricongiungimento con Traiano.

$98 \mathrm{Su}$ Antiochia di Pisidia cfr. Drew-Bear - Tașhalan - Thomas 2002.

99 AE 1926, 81 = 1928, 101, Konya / Iconium: Clodiae Iatr/inae uxori L(uci) / Cossoni Galli / leg(ati) Aug(usti) pr(o) pr(aetore) / Eburena Ma/xima C(ai) Ebure/[ni - - -]. L'iscrizione è considerata funeraria (Grabinschrift) nella Epigraphische Datenbank zum antiken Kleinasien (http://www.epigraphik.uni-hamburg. de/datenbank/suche?id=2054), ma benché frammentaria, non appare plausibile che a porre un epitafio fosse una amica e non il marito vivente. Cfr. Ramsay 1941, 135, nr. 143; McLean 2007. Il raro nome Eburenus è di origine celtica (galata) (cfr. StÄhelin 1907, 114-115; Mitchell 1979, 409-438; SAlomies 1997, 246, n. 10).

100 RAMSAY 1926, 214-215, nr. 6; HoRnUm 1993, 294, nr. 245.

101 ECK 2013.

102 Thomasson 2009, 133, nr. 34:026 a “(1) L. Cossonius Gallus Vecilius Crispinus Mansuanius Marcellinus Numisius Sabinus, cos. 116 (cf. $P I R^{2}$ G 71; RE I 2268 sq., n. 51; DNP 12/2, 934). - Primis annis Hadriani. - [leg. imp. Hadriani Aug. p]r. pr. [p]rovin[ciae Iudaeae] The Israel Academy of Sciences and Humanities. Proceedings, VII: 7. 2001, pp. 219-223, n. 1 = AE 2003: 1801, Caesarea mar. Nomina etsi non nisi partim servata cos. a. 116 apta sunt, quem ante consulatum alteram provinciam imperatoris regere potuisse negant Hannah M. Cotton - W. Eck (Proceedings, cit.) de legato Iudaeae consulari cogitantes".
} 


\section{Conclusioni}

La carriera di L(ucius) Cossonius Gallus appare segnata dal suo rapporto personale con Traiano, presumibilmente maturato durante gli incarichi di legatus provinciae Asiae e di quaestor della provincia di Pontus et Bithynia. La relazione con Traiano dovette essere cementata durante il bellum dacicum al quale Cossonio partecipò come legatus della legio I Italica. Successivamente Cossonio fu legatus della legio II Traiana Fortis. Fu Traiano a ricostituire la provincia proconsolare della Sardinia, affidando allo stesso Cossonio il ruolo di proconsul Sardiniae con l'incarico della constitutio di Forum Traiani, probabilmente nel 111. Nel corso della carica di legatus Augusti propraetore della Galatia Lucius Cossonius Gallus (113-115) ebbe un ruolo organizzativo delle truppe per la campagna traianea contro l'Armenia e nel 116 Cossonio raggiunse il rango di consul suffectus. Dopo la morte di Traiano la carriera di Cossonio proseguì sotto Adriano raggiungendo la carica di legatus consolare della Iudaea.

\section{BIBLIOGRAFIA}

ALFÖLDY, G.

(1992): Studi sull'epigrafia augustea e tiberiana di Roma (=Vetera 8), Roma.

(1999): "Die Inschriften des Jüngeren Plinius und seine Mission in Pontus et Bithynia", AAntHung 39, 1999, 21-44.

(1999a): Städte, Eliten und Gesellschaften in der Gallia Cisalpina (=Heidelberger Althistorische Beiträge und Epigraphische Studien 30), Stuttgart.

Angeli Bertinelli, M. G. (2000): “Traiano in Oriente: la conquista dell'Armenia, della Mesopotamia e dell'Assiria", [in] González (ed.), 2000, 12-35.

Angiolillo, S. (1981): Mosaici antichi in Italia. Sardinia, Roma.

Atzori, S. (2006): La strada romana a “Karalibus Svlcos”, Mogoro.

BAcco, G. - Ganga, T. - Oppo, Ch. - Serra, P. B. - VACCA, M. - Zanella, R. M. - Zucca, R. (2010): "Structores amphitheatri. A proposito dell'anfiteatro di Forum Traiani (Sardinia)", [in] M. Milanese - P. Ruggieri - C. Vismara (curs.), L'Africa romana XVIII. I luoghi e le forme dei mestieri e della produzione nelle province africane, Roma, vol. II, 1371-1460.

BAille, L. (1797): Sigillo de 'Bassi tempi illustrato, Torino.

BARresi, P. (2003): Province dell'Asia Minore: Costo dei marmi, architettura pubblica e committenza, Roma.

Bennett, J. (2005): Trajan Optimus Princeps. A Life and Times, London-New York (1 ${ }^{\mathrm{a}}$ ed. 1997, London).

BÉRARD, F.

(1984): "La carrière de Plotius Grypus et le ravitaillement de l'armée impériale en campagne", MEFRA 96/1, 259-324.

(2000): "La légion XXI ${ }^{\mathrm{e}}$ Rapax" [in] Y. Le Bohec - C. Wolff (éd.), Les Légions de Rome sous le Haut-Empire. Actes du Congrès de Lyon (17- 19 septembre 1998), Lyon, 49-67. 
Brandt, H. (1992): Geschichte und Wirtschaft Pamphyliens und Pisidiens im Altertum (=Asia Minor Studien 7), Bonn.

Camodeca, G. (1982): “Ascesa al Senato e rapporti con i territori d'origine. Italia: regio I (Campania) e le regiones II e III”, [in] Epigrafia e ordine senatorio Atti del colloquio internazionale AIEGL (Roma 14-20 maggio 1981), (=Tituli 5), Roma, vol. II, 101-163.

Campos Carrasco, J. M. - Pérez Macías, J. A. (2003): “Los programas edilicios de época trajana", [en] J. Alvar - J. Ma Blázquez (eds.), Trajano, Madrid, 269-300.

Cascione, C. (1999): Tresviri capitales. Storia di una magistratura minore (=Pubblicazioni del Dipartimento di Diritto Romano e Storia della Scienza Romanistica dell'Università degli Studi di Napoli Federico II 13), Napoli.

Castagna, L. - Lefèvre, E. (Hg.), (2003): Plinius der Jüngere und seine Zeit, MünchenLeipzig.

Casula, F. C. (2006): Dizionario storico sardo VII, Sassari.

Cébeillac-Gervasoni, M. (1982): “Ascesa al senato e rapporti con i territori d'origine. Italia: regio I (Campania: la zona di Capua e Cales)" [in] Epigrafia e ordine senatorio Atti del colloquio internazionale AIEGL (Roma 14-20 maggio 1981), (=Tituli 5), Roma, vol. II, 59-99.

Crespo Cabillo, C. (2009): "Los fora de época imperial: los ejemplos alpinos y sardos", Veleia 26, 287-295.

Cristofori, A. (2004): Non arma virumque. Le occupazioni nell'epigrafia del Piceno, Bologna.

DieTz, K. (1980): Senatus contra principem. Untersuchungen zur senatorischen Opposition gegen Kaiser Maximinus Thrax, München.

Drew-Bear, Th. - Taşhalan, M. - Thomas, Ch. M. (ÉD.), (2002): Actes du Ier Congrès International sur Antioche de Pisidie (=Collection Archéologie et Histoire de l'Antiquité, Lyon 5), Lyon-Paris.

ECK, W.

(1970): Senatoren von Vespasian bis Hadrian. Prosopographische Untersuchungen mit Einschlu $\beta$ der Jahres- und Provinzialfasten der Statthalter (=Vestigia 13), München.

(2001): "Die große Pliniusinschrift aus Comum: Funktion und Monument", [in] A. Bertinelli - A. Donati (eds.), Varia Epigraphica. Atti del Colloquio Internazionale di Epigrafia, Bertinoro, 8-10 giugno 2000, Faenza, 225-235.

(2005): "Auf der Suche nach Personen und Persönlichkeiten: Cursus honorum und Biographie, in Biographie und Prosopographie", [in] K. Vössing (hg.), Festschrift zum 65. Geburtstag von Anthony R. Birley, Stuttgart, 53-72.

(2007): "Die politisch-administrative Struktur der kleinasiatischen Provinzen während der hohen Kaiserzeit", [in] G. Urso (ed.), Tra Oriente e Occidente. Indigeni, Greci e Romani in Asia Minore. Atti del convegno internazionale, Cividale del Friuli, 28-30 settembre 2006, (=I convegni della Fondazione Niccolò Canussio 6), Pisa, 189-207.

(2009): "There Are No cursus honorum Inscriptions. The Function of the cursus honorum in Epigraphic Communication", Scripta Classica Israelica 28, 79-92.

(2013): “Cossonius", [in] Brill's New Pauly. Brill Online, 2013 (http://referenceworks.brillonline.com/entries/brill-s-new-pauly/cossonius-e12220910).

(2013a): "Mansuanius", [in] Brill's New Pauly. Brill Online, 2013 (http://referenceworks. brillonline.com/entries/brill-s-new-pauly/mansuanius-e12223820).

(2013b): “Catilius", [in] Brill's New Pauly. Brill Online, 2013 (http://referenceworks.brillonline.com/entries/brill-s-new-pauly/catilius-e229020). 
Eck, W. - HeIL, M. (HG.), (2005): Senatores populi Romani. Realität und mediale Präsentation einer Führungsschicht (=HABES 40), Stuttgart.

FAoro, D. (2011): Praefectus, procurator, praeses. Genesi delle cariche presidiali equestri nell'Alto Impero Romano, Firenze.

Feraudi-GruénAis, F. (2005): "Für die Ewigkeit? Die Gestaltung von senatorischen Grablegen Roms und ihr Kontext”, [in] Eck - Heil (hg.), 2005, 137-168.

FÉvrier, J. G. (1946-1949): "Les Phéniciens et la Sardaigne”, BCTH (février 1948), 415-419.

French, D. H. (2003): Roman, Late Roman and Byzantine Inscriptions of Ankara. A Selection, Ankara.

Galliazzo, V. (1995): I ponti romani. II. Catalogo generale, Treviso.

GARRUCCI, R. (1864): Della Via Clodia e coerenti e delle città e villaggi che furono sul corso di esse (=Dissertazioni archeologiche di vario argomento I), Roma.

GASPERINI, L. (1996): “Olbiensia epigraphica”, [in] A. Mastino - P. Ruggeri (curs.), Da Olbia ad Olbia, 2500 anni di una città mediterranea. Atti del Convegno maggio 1994. I, Olbia in età antica, Sassari, 311-316.

Gнiотто, A. R. (2004): L'architettura romana nelle città della Sardegna (=Antenor Quaderni 4), Roma.

GonzÁlez, J. (ED.), (2000): Trajano emperador de Roma (=Saggi di Storia Antica 16), Roma.

Gros, P. (2000): “La «militarisation» de l'urbanisme trajanien à la lumière des recherches récentes sur le Forum Traiani”, [in] González (ed.), 2000, 227-249.

Guey, J. (1937): Essai sur la guerre parthique de Trajan (114-117), (=Bibliothèque d'Istros 2), Bucarest.

Guzzo Amadasi, M. G. (1967): Le iscrizioni fenicie e puniche delle colonie in Occidente (=Studi Semitici 28), Roma.

HARRIS, W. V.

(1965): "The Via Cassia and the Via Traiana Nova between Bolsena and Chiusi", PSBR 33, 113-133 (http://dx.doi.org/10.1017/S0068246200007352).

(1991): “A Milestone from the Via Traiana Nova near Orvieto (AE 1969/1970, 186a and b)", ZPE 85, 186-188.

Hillebrand, S. (2006): Der Vigintivirat: Prosopographische Untersuchungen für die Zeit von Augustus bis Domitian, Dissertation, Ruprecht-Karls-Universität Heidelberg Zentrum für Altertumswissenschaft Seminar für Alte Geschichte und Epigraphik.

Hornum, M. B. (1993): Nemesis, the Roman State and the Games, London.

IвBA, A. (2009): “L’esercito e la flotta”, [in] Mastino (cur.), 2009, 393-404.

JACQUES, F. (1983): Les curateurs des cités dans l'Occident romain: de Trajan à Gallien (=Études prosopographiques V), Paris.

Jones, B. W. (2002): The Emperor Domitian, London-New York (1 ${ }^{\text {a }}$ ed. 1992).

Le Bohec, Y. (1990): La Sardaigne et l'armée romaine sous le Haut-Empire, Sassari.

LePPer, F. A. (1948): Trajan's Parthian War, Oxford.

Levi della Vida, G. (1934-1935): "L’iscrizione punica di Bitia in Sardegna”, Atti della R. Accademia delle Scienze di Torino 70, 185-198. 
LORETO, L. (2000): Il comando militare nelle province procuratorie 30 a.C. - 280 d.C. Dimensione militare e dimensione costituzionale (=Pubblicazioni della Facoltà di Giurisprudenza della Seconda Università di Napoli 12), Napoli.

Luzzatto, G. I. (1985): Roma e le province I, Bologna.

MAREK, $\mathrm{CH}$.

(1993): Stadt, Ära und Territorium in Pontus-Bithynia und Nord-Galatia (=Istanbuler Forschungen 39), Tübingen.

(2003): Pontus et Bithynia. Die Römischen Provinzen im Norden Kleinasiens (=Orbis provinciarum VI, 199), Mainz.

(2013): "Paphlagonia", [in] Brill's New Pauly. Brill Online (http://referenceworks. brillonline.com/entries/brill-s-new-pauly/paphlagonia-e906690).

Mastino, A. (1986): "Supplemento epigrafico turritano", Nuovo bullettino archeologico sardo $3,189-205$.

Mastino, A. (Cur.), (2009): Storia della Sardegna antica, Nuoro².

Mastino, A. - Ruggeri, P. (1998): "I falsi epigrafici romani delle Carte d'Arborea", [in] L. Marrocu (ed.), Le Carte d'Arborea. Falsi e falsari nella Sardegna del XIX secolo. Atti del Convegno "Le Carte d'Arborea”, Oristano 22-23 marzo 1996, Cagliari, 219-274.

Mastino, A. - Zucca, R. (c.s.): "Un nuovo titulus della cohors Ligurum in Sardinia e il problema dell'organizzazione militare della Sardegna nel I secolo d.C.”, [in] AA.VV., L'iscrizione e il suo doppio. Atti del Colloquio internazionale di epigrafia (Bertinoro, Centro Residenziale Universitario. Colloquio Borghesi; 6-8 giugno 2013).

McAlindon, D. (1957): "Entry to the Senate in the Early Empire", JRS 47, 191-193 (http:// dx.doi.org/10.2307/298584).

McLean, B. H. (2007): Greek and Latin Inscriptions in the Konya Archaeological Museum, Ankara.

Meloni, P. (1958): L'amministrazione della Sardegna da Augusto all'invasione vandalica, Roma.

Migliorati, G. (2003): Cassio Dione e l'impero romano da Nerva ad Antonino Pio. Alla luce dei nuovi documenti, Milano.

Mitchell, S.

(1979): "Iconium and Ninica: Two double communities in Roman Asia Minor", Historia 28, 409-438.

(1993): Anatolia. Land, men and gods in Asia Minor. Vol. I. The Celts and the impact of Roman Rule, Oxford.

Mitchell, S. - French, D. (2012): The Greek and Latin Inscriptions of Ankara (Ancyra), Vol. I: From Augustus to the end of the third century AD (=Vestigia 62), München.

Mócsy, A. (1974): Pannonia and Upper Moesia. A History of the Middle Danube Provinces of the Roman Empire, London-Boston.

Moretti, E. (1925): La Via Cassia e la Via Traiana Nova a Volsinis ad Fines Clusinorum, Orvieto.

Oliver, J. H. (1947): “The descendants of Asinius Pollio", AJPh 68, 147-160 (http://dx.doi. org/10.2307/290950). 
PAIS, E.

(1894): "Prima relazione intorno ai viaggi fatti per la compilazione dei Supplemento Italica al Corpus Inscriptionum Latinarum", Rendiconti della Reale Accademia dei Lincei, Cl. Sc. Morali, Storiche e Filologiche, ser. V, 907-929.

(1999): Storia della Sardegna e della Corsica durante il dominio romano, Nuoro ( $1^{\mathrm{a}}$ ed. Roma 1923, A. Mastino, cur.).

Pala, P. - SAnciu, A. - SAnges, M. (2013): “Un nuovo diploma militare dalla Sardegna”, ZPE 186, 301-306.

Paulis, G. (1987): I nomi di luogo della Sardegna, Sassari.

RAMSAY, W. M.

(1926): "Studies in the roman province of Galatia", JRS 16, 214-215 (http://dx.doi. org/10.2307/296186).

(1941): The social basis of Roman power in Asia Minor, Aberdeen.

RÉMY, B.

(1986): L'évolution administrative de l'Anatolie aux trois premiers siècles de notre ère (=Collection du Centre d'Études Romaines et Gallo-Romaines 5), Lyon.

(1989): Les carrières sénatoriales dans les provinces romaines d'Anatolie au Haut-Empire (31 av. J.-C.-284 ap. J.-C.): Pont-Bithynie, Galatie, Cappadoce, Lycie-Pamphylie et Cilicie (=Varia Anatolica 2), Istambul.

RitTERLING, E.

(1893): "Zur römischen Legionsgeschichte am Rhein. II. Der Aufstand des Antonius Saturninus", Westdeutsche Zeitschrift für Geschichte und Kunst 12, 203-242.

(1925): "Legio I Italica", [in] RE XII, 1407-1417.

(1925a): "Legio XXI Rapax”, [in] RE XII, 1736, 1788-1789.

RÜPke, J. - Glock, A. (2005): Fasti sacerdotum, Die Mitglieder der Priesterschaften und das sakrale Funktionspersonal römischer, griechischer, orientalischer und jüdisch-christlicher Kulte in der Stadt Rom von 300 v. Chr bis 499 n. Chr., Teil 2: Biographien, Stuttgart.

Salomies, O. (1997): "Die Herkunft des Numidischen Legaten Ti. Claudius Subatianus Proculus", ZPE 119, 245-248.

SARnowski, T. (2001): "Headquarters building (principia) of legio I Italica at Novae", [in] P. Dyczek (ed.), Novae - 40 years of excavations, Warszawa, 31-37.

SETTIPANi, CH.

(2000): Continuité gentilice et continuité familiale dans les familles senatoriales romaines à l'époque imperiale. Mythe et realité (=Occasional publications of the Unit for Prosopographical Research; Prosopographica et Genealogica 2), Oxford.

(2002): Addenda I - III (juillet 2000- octobre 2002), Prosopographica et Genealogica, s.1.

SHERK, R. K.

(1951): The legates of Galatia from Augustus to Diocletian (=The John's Hopkins University Studies in historical and political science 69/2), Baltimore.

(1979): "A chronology of the governors of Galatia, A. D. 112-285", AJPh 100, 166-175.

(1980): "Roman Galatia: the governors from 25 B.C. to A.D. 114", $A N R W$ II 7.2, 954-960.

SHERwin-White, A. N. (1966): The Letters of Pliny: A Historical and Social Commentary, Oxford.

SisANI, S. (2011): “In pagis forisque et conciliabulis. Le strutture amministrative dei distretti rurali in Italia tra la media Repubblica e l'età municipale", Memorie dell'Acc. Naz. Dei Lincei, Classe di Scienze Morali, Storiche e Filologiche IX, v. 21, 543-780. 
Spano, G. (1874): Scoperte archeologiche fattesi in Sardegna in tutto l'anno 1874, Cagliari.

StÄHELIN, F. (1907): Geschichte der Kleinasiatischen Galater, Leipzig (2ª ed.).

Strobel, K.

(1986): "Der Aufstand des L. Antonius Saturninus und der sogenannte zweite Chattenkrieg Domitians", Tyche 1, 203-220.

(1988): "Zur Dislozierung der römischen Legionen în Pannonien zwischen 89 und 118 n. Chr.", Tyche III, 193-222.

(1988a): "Zu Fragen der frühen Geschichte der römischen Provinz Arabia und zu einigen Problemen der Legionsdislokation im Osten des Imperium Romanum zu Beginn des 2. Jh. n. Chr.", ZPE 71, 251-280.

(1989): Die Donaukriege Domitians (=Antiquitas 38), Bonn.

(1998): Die Galater, Berlin.

(2013): "Bithynia et Pontus", [in] Brill's New Pauly. Brill Online, (http://referenceworks. brillonline.com/entries/brill-s-new-pauly/bithynia-et-pontus-e217940).

(2013a): “Cappadocia, II”, [in] Brill's New Pauly. Brill Online, (http://referenceworks.brillonline.com/entries/brill-s-new-pauly/cappadocia-ii-e226730).

(2013b): "Galatia", [in] Brill's New Pauly. Brill Online, (http://referenceworks.brillonline. com/entries/brill-s-new-pauly/galatia-e417820).

SYME, R.

(1936): "Flavian wars and frontiers", [in] S. A. Cook - F. E. Adcock - M. P. Charlesworth (eds.), Cambridge Ancient History, Cambridge, vol. XI, 131-187.

(1938): “The First Garrison of Trajan's Dacia”, Laurea Aquincenses I, 267-286 [=Danubian Papers, Bucureşti, 1971].

Teja, R. (1980): "Die römische Provinz Kappadokia in der Prinzipatszeit", $A N R W$ II 7.2, 1083-1124.

Thomasson, B. E. (2009): Laterculi Praesidum, I ex parte retractatum, Göteborg.

Todisco, E. (2013): I vici rurali nel paesaggio dell'Italia romana (=Documenti e studi 50), Bari.

Tola, P. (1861): Codex diplomaticus Sardiniae, Torino, vol. I.

Torelli, M. (1982): "Italia, regio VII (Etruria)", [in] Epigrafia e ordine senatorio Atti del colloquio internazionale AIEGL (Roma 14-20 maggio 1981), (=Tituli 5), Roma, vol. II, 275-299.

Torelli, M. R. (2002): Benevento romana (=Saggi di storia antica 18), Roma.

UGGERI, G. (1983): La viabilità romana nel Salento (=Testi e monumenti del Museo Archeologico 'Ugo Granafei’ di Mesagne 4), Mesagne.

Urloiu, R. (2010): “Legio II Traiana Fortis and Judaea under Hadrian's Reign”, Cogito 4, s.p. (http://cogito.ucdc.ro/n4e/LEGIO-II-TRAIANA-FORTIS-AND-JUDAEA-UNDER-HADRIAN-REIGN.pdf).

VAglieri, D. (1910): “Galatia”, [in] E. De Ruggiero, Dizionario Epigrafico di Antichità Romane, Roma, vol. III, 361-363.

VAlvo, A. (2007): "Origine e provenienza delle gentes italiche nella provincia di Galazia in età giulio-claudia”, [in] G. Urso (ed.), Tra Oriente e Occidente. Indigeni, Greci e Romani in Asia minore. Atti del convegno internazionale, Cividale del Friuli, 28-30 settembre 2006, Pisa, 151-163. 
Wehmann, Рн. (2008): Eine römische Statthalterschaft - Plinius der Jüngere in Pontus et Bithynia, Dresden.

Wilkes, J. J. (1983): "Romans, Dacians and Sarmatians in the First and Early Second Centuries", [in] B. Hartley - J. Wacher (eds.), Rome and her Northern provinces. Papers presented to Sheppard Frere, Oxford, 269-270.

Williams, W. (1990): Pliny: Correspondence with Trajan from Bithynia (Epistles X), Warminster.

ZedDA, F. (1906): Forum Traiani, Roma.

ZuCCA, R.

(1994): "Il decoro urbano delle civitates Sardiniae et Corsicae: il contributo delle fonti letterarie ed epigrafiche", [in] A. Mastino - P. Ruggeri (curs.), L'Africa Romana. Atti del X convegno di studio (Oristano 11-13 dicembre 1992), Sassari, 857-935.

(2001): “Additamenta epigraphica all'amministrazione della Sardegna da Augusto all'invasione vandalica", [in] G. Angelli Bertinelli - A. Donati (curs.), Varia epigraphica. Atti del Colloquio internazionale di epigrafia (Bertinoro 8-10 giugno 2000), (=Epigrafia e antichità 17), Faenza, 516-527.

(2009): "L'urbanistica di Forum Traiani”, [in] C. Marangio - G. Laudizi (curs.), Studi di Topografia antica in onore di Giovanni Uggeri, Galatina, 573-586. 